\title{
Microlocal Parametrices for Hyperbolic Mixed Problems in the Case Where Boundary Waves Appear
}

\author{
By
}

\author{
Seiichiro WAKABAYASHI*
}

\section{§1. Introduction}

In this paper we shall construct microlocal parametrices for hyperbolic mixed problems at non-glancing points in the case where their Lopatinski's determinants have real zeros, and we shall investigate the reflection of singularities. It was proved for hyperbolic mixed problems with constant coefficients in a quarter-space that singularities corresponding to boundary waves generally appear when Lopatinski's determinant has real zeros (see [2], [11], [12]). We shall show that singularities corresponding to boundary waves appear in variable coefficients cases.

Microlocal parametrices for hyperbolic mixed problems were constructed in some cases by using the theory of Fourier integral operators. Chazarain [1] constructed microlocal parametrices for the Dirichlet problem for wave equations at non-glancing points. Microlocal parametrices for the Dirichlet problem for second order operators were constructed at diffractive points by Melrose [6] and Taylor [10]. But it seems very difficult to construct microlocal parametrices at glancing points which are not diffractive. On the other hand there is a problem of constructing microlocal parametrices when Lopatinski's determinant has real zeros. This problem has no difficulty and we can investigate the reflection of singularities corresponding to boundary waves by constructing microlocal parametrices. We can construct a microlocal parametrix as the

* Communicated by S. Matsuura, July 23, 1976.

Department of Mathematics, University of Tsukuba, Ibaraki 300-31, Japan. 
composition of a microlocal parametrix for the Dirichlet problem and a microlocal parametrix for the Cauchy problem for a system of pseudo-differential operators on the boundary when Lopatinski's determinant has real zeros (see §5). However, we shall construct microlocal parametrices more directly in that case.

Now let us state our problem and assumptions. Let $\boldsymbol{R}^{n+1}$ denote the $(\mathrm{n}+1)$-dimensional Euclidean space and write $x^{\prime}=\left(x_{1}, \cdots, x_{n}\right)$, $x^{\prime \prime}=\left(x_{2}, \cdots, x_{n}\right)$ for the coordinate $x=\left(x_{0}, x_{1}, \cdots, x_{n}\right)$ in $\mathbb{R}^{n+1}$ and $\xi^{\prime}=\left(\xi_{1}, \cdots, \xi_{n}\right), \quad \xi^{\prime \prime}=\left(\xi_{2}, \cdots, \xi_{n}\right)$ for the dual coordinate $\xi=\left(\xi_{0}, \cdots\right.$, $\left.\boldsymbol{\xi}_{n}\right)$ in $\boldsymbol{R}^{n+1}$. We shall also denote by $\boldsymbol{R}_{+}^{n+1}$ the half-space $\left\{x=\left(x_{0}\right.\right.$, $\left.\left.x^{\prime}\right) \in \mathbb{R}^{n+1} ; x_{0}>0\right\}$ and use the symbol $D=i^{-1}\left(\partial / \partial x_{0}, \cdots, \partial / \partial x_{n}\right)$. Let $P(x, \xi)$ be a polynomial of order $m$ of $n+1$ variables $\xi$ with $C^{\infty}$ coefficients and $p(x, \xi)$ its principal part. We assume that $p(x, \xi)$ is a strictly hyperbolic polynomial with respect to $\xi_{1}$ and $p(x, 1,0$, $\cdots, 0)=1$. Thus we can write

$$
p(x, \xi)=\prod_{j=1}^{l}\left(\xi_{0}-\lambda_{j}^{+}\left(x, \xi^{\prime}\right)\right) \cdot \prod_{j=1}^{m-l}\left(\xi_{0}-\lambda_{j}^{-}\left(x, \xi^{\prime}\right)\right),
$$

where the $\lambda_{j}^{ \pm}\left(x, \xi^{\prime}\right)$ are continuous in $\left(x, \xi^{\prime}\right)$ and

$$
\operatorname{Im} \lambda_{j}^{ \pm}\left(x, \xi^{\prime}\right) \gtrless 0 \text { when } \operatorname{Im} \xi_{1}<0, \xi^{\prime \prime} \in \boldsymbol{R}^{n-1} .
$$

We consider the mixed initial-boundary value problem for the hyperbolic operator $P(x, D)$ in a quarter-space

$$
\begin{aligned}
& P(x, D) u(x)=f(x), \quad x \in \boldsymbol{R}_{+}^{n+1}, x_{1}>0, \\
& \left.D_{1}^{k-1} u(x)\right|_{x_{1}=0}=0, \quad x_{0}>0, \quad 1 \leqq k \leqq m, \\
& \left.B_{j}\left(x^{\prime}, D\right) u(x)\right|_{x_{0}=0}=g_{j}\left(x^{\prime}\right), \quad x_{1}>0, \quad 1 \leqq j \leqq l .
\end{aligned}
$$

Here the $B_{j}\left(x^{\prime}, D\right)$ are boundary operators with $C^{\infty}$ coefficients.

Now let $\left(x^{0^{\prime}}, \xi^{0^{\prime}}\right)$ be a fixed point in $T^{*} \boldsymbol{R}^{n} \backslash 0$ and put $x^{0}=\left(0, x^{0^{\prime}}\right)$. We may assume that the $\lambda_{j}^{+}\left(x, \xi^{\prime}\right)$ are enumerated in the following way:

$$
\begin{array}{ll}
\operatorname{Im} \lambda_{j}^{+}\left(x^{0}, \quad \xi^{\prime}\right)=0 & \text { for } 1 \leqq j \leqq l_{1}, \\
\operatorname{Im} \lambda_{j}^{+}\left(x^{0}, \quad \xi^{\prime}\right)>0 & \text { for } l_{1}+1 \leqq j \leqq l .
\end{array}
$$

Then we put

$$
\begin{aligned}
& L\left(x^{\prime}, \xi^{\prime}\right)=\left(b_{j}\left(x^{\prime}, \lambda_{1}^{+}\left(0, x^{\prime}, \xi^{\prime}\right), \xi^{\prime}\right), \cdots, b_{j}\left(x^{\prime}, \lambda_{l_{1}^{\prime}}^{+}, \xi^{\prime}\right),\right. \\
& \left.\frac{1}{2 \pi i} \int_{c_{\xi^{\prime}}} \frac{b_{j}\left(x^{\prime}, \xi\right)}{p\left(0, x^{\prime}, \xi\right)} d \xi_{0}, \cdots, \frac{1}{2 \pi i} \int_{c_{\xi^{\prime}}} \frac{b_{j}\left(x^{\prime}, \xi\right) \xi_{0}^{l-l_{1-1}-1}}{p\left(0, x^{\prime}, \xi\right)} d \xi_{0}\right)_{j \downarrow 1, \ldots l l},
\end{aligned}
$$


where $b_{j}\left(x^{\prime}, \xi\right)$ is the principal part of $B_{j}\left(x^{\prime}, \xi\right)$ and $C_{\xi^{\prime}}$ is a simple closed curve enclosing only roots $\lambda_{l_{1}+1}^{+}\left(0, x^{\prime}, \xi^{\prime}\right), \cdots, \lambda_{l}^{+}\left(0, x^{\prime}, \xi^{\prime}\right)$ of $p\left(0, x^{\prime}, \lambda, \xi^{\prime}\right)=0$, and 'we define Lopatinski's determinant ${ }^{\dagger}$ for $\left\{p, b_{j}\right\}$ by

$$
R\left(x^{\prime}, \xi^{\prime}\right)=\operatorname{det} L\left(x^{\prime}, \xi^{\prime}\right) .
$$

Remark. It is easy to see that

$$
\begin{aligned}
R\left(x^{\prime}, \xi^{\prime}\right)= & (-1)^{l_{1}\left(l-l_{1}\right)} \Pi_{1 \leq j<k \leq l_{1}}\left(\lambda_{j}^{+}\left(0, x^{\prime}, \xi^{\prime}\right)-\lambda_{k}^{+}\right) / \prod_{\substack{l_{1}+1 \leq j \leq l \\
1 \leq k \leq m-l}}\left(\lambda_{j}^{+}-\lambda_{k}^{-}\right) \\
& \times \operatorname{det}\left(\frac{1}{2 \pi i} \oint \frac{b_{k}\left(x^{\prime}, \xi\right) \xi_{0}^{j-1}}{p_{+}\left(0, x^{\prime}, \xi\right)} d \xi_{0}\right)_{j, k=1, \ldots, l},
\end{aligned}
$$

where $p_{+}(x, \xi)=\Pi_{j=1}^{l}\left(\xi_{0}-\lambda_{j}^{+}\left(x, \xi^{\prime}\right)\right)$.

We state the assumptions that we impose on $\left\{p, b_{j}\right\}$ :

(A. 1) $\left(x^{0}, \xi^{\prime \prime}\right)$ is not a glancing point for $p$, i.e., $\lambda_{j}^{+}\left(x^{0}, \xi^{0^{\prime}}\right), 1 \leqq j$ $\leqq l_{1}$, are simple real roots of $p\left(x^{0}, \lambda, \xi^{0}\right)=0$.

$$
\text { (A. 2) } \quad R\left(x^{\prime}, \xi^{\prime}\right)=\left(\xi_{1}-\xi_{1}\left(x^{\prime}, \xi^{\prime \prime}\right)\right)^{\theta} r\left(x^{\prime}, \xi^{\prime}\right) \text {, }
$$

where $\xi_{1}\left(x^{\prime}, \xi^{\prime \prime}\right)$ and $r\left(x^{\prime}, \xi^{\prime}\right)$ are $C^{\infty}$ functions defined in a conic neighborhood of $\left(x^{0^{\prime}}, \xi^{\prime}\right)$ in $T^{*} \boldsymbol{R}^{n} \backslash 0, \xi_{1}\left(x^{\prime}, \xi^{\prime \prime}\right)$ is real valued and homogeneous of degree 1 in $\xi^{\prime \prime}, \xi_{1}\left(x^{0^{\prime}}, \xi^{0 \prime \prime}\right)=\xi_{1}^{0}, r\left(x^{0^{\prime}}, \xi^{\prime \prime}\right) \neq 0$ and $\theta$ is a positive integer.

(A. 3) There exist $l \times l$ matrix valued $C^{\infty}$ functions $U\left(x^{\prime}, \xi^{\prime}\right)$ and $V\left(x^{\prime}, \xi^{\prime}\right)$ defined in a conic neighborhood of $\left(x^{0^{\prime}}, \xi^{0^{\prime}}\right)$ in $T^{*} \mathbb{R}^{n} \backslash 0$ such that

$$
U\left(x^{\prime}, \xi^{\prime}\right) L\left(x^{\prime}, \xi^{\prime}\right) V\left(x^{\prime}, \xi^{\prime}\right)=\left[\begin{array}{cc}
\left(\xi_{1}-\xi_{1}\left(x^{\prime}, \xi^{\prime \prime}\right)\right) I_{\theta^{\prime}} & 0 \\
0 & L_{c}\left(x^{\prime}, \xi^{\prime}\right)
\end{array}\right],
$$

$\operatorname{det} U\left(x^{0^{\prime}}, \xi^{0^{\prime}}\right) \neq 0$, det $L_{e}\left(x^{0^{\prime}}, \xi^{0^{\prime}}\right) \neq 0$, the $(i, j)$-entry of $U$ is homogeneous of degree $1-\rho_{i}-m_{j}$ and the $(i, j)$-entry of $V$ is homogeneous of degree $\rho_{j}$ for $1 \leqq i \leqq l_{1}$ and of degree $\rho_{j}+m+l_{1}-i$ for $l_{1}+1 \leqq i \leqq l$, where $\theta^{\prime}$ is a positive integer, $I_{\theta^{\prime}}$ is the identity matrix of order $\theta^{\prime}$, $L_{e}$ is an $\left(l-\theta^{\prime}\right) \times\left(l-\theta^{\prime}\right)$ matrix and $\operatorname{deg} B_{j}=m_{j}$.

Remark. (i) If the condition (A. 2) with $\theta=1$ is satisfied then the condition (A. 3) also holds. In fact, taking $U\left(x^{\prime}, \xi^{\prime}\right)=I$ and

$\dagger$ Lopatinski's determinant defined above is different from original one. 
$V\left(x^{\prime}, \xi^{\prime}\right)=r\left(x^{\prime}, \xi^{\prime}\right)^{-1}$ 'cof $L\left(x^{\prime}, \xi^{\prime}\right)$, we have $U L V=\left(\xi_{1}-\xi_{1}\left(x^{\prime}, \xi^{\prime \prime}\right)\right) I$. (ii) Suppose that $\lambda_{j}^{+}\left(x^{0}, \xi^{\prime \prime}\right), 1 \leqq j \leqq l_{2}$, are simple roots of $p\left(x^{0}, \lambda, \xi^{0^{\prime}}\right)$ $=0$. If $\operatorname{rank}\left(B_{j}\left(x^{0}, \lambda_{k}^{+}\left(x^{0}, \xi^{\prime}\right), \xi^{0 \prime}\right)\right)_{\substack{j, 1 \\ k \rightarrow 1, \ldots, l}, l_{2}}=l_{2}-\theta$ the condition (A. 3) follows from (A. 2) (see [4]).

Let $\Gamma$ be a conic neighborhood of $\left(x^{0^{\prime}}, \xi^{\prime \prime}\right)$ in $T^{*} \mathbb{R}^{n} \backslash 0$ and $U$ a neighborhood of $x^{0^{\prime}}$ in $\mathbb{R}^{n}$. Let us define a microlocal parametrix for the problem

(1. 1)' $\quad P(x, D) u(x)=0, \quad x \in \mathbb{R}_{+}^{n+1}, \quad x_{1}>0$,

(1.2)' $\left.\quad D_{1}^{j-1} u(x)\right|_{x_{1}=0}=0, \quad x_{0}>0, \quad 1 \leqq j \leqq m$,

$\left.(1.3)^{\prime} \quad B_{j}\left(x^{\prime}, D\right) u(x)\right|_{x_{0}=0}=\delta_{j k} g\left(x^{\prime}\right), \quad x_{1}>0, \quad 1 \leqq j \leqq l$,

where $1 \leqq k \leqq l$.

Definition 1. 1. A right microlocal parametrix (Poisson operator) for the problem $(1.1)^{\prime}-(1.3)^{\prime}$ at $\left(x^{0}, \xi^{0^{\prime}}\right)$ is a triple $\left\{E_{k}, \Gamma,[0, \varepsilon) \times U\right\}$ satisfying the conditions

(i) $E_{k}$ is a continuous linear map: $\mathscr{D}^{\prime}(U) \rightarrow C^{\infty}\left([0, \varepsilon) ; \mathscr{D}^{\prime}(U)\right) \dagger$,

(ii) $P E_{k}(g) \in C^{\infty}([0, \varepsilon) \times U)$,

(iii) $\left.B_{j} E_{k}(g)\right|_{x_{0}=0}-\delta_{j k} g \in C^{\infty}(U), \quad 1 \leqq j \leqq l$, if $W F(g) \subset \Gamma$,

(iv) $\left.E_{k}(g)\right|_{x_{1}<c}$ is smooth if $W F(g) \subset\left\{x_{1} \geqq c\right\}$.

The remainder of this paper is organized as follows. In $\S 2$ we shall formally construct a microlocal parametrix. In $\S 3$ the procedure of $\S 2$ will be justified and singularities of a microlocal parametrix will be studied. In $\S 4$ we shall construct microlocal parametrices for the problem (1.1)-(1.3), following Melrose [6], and study reflection of singularities of solutions to the problem (1.1)-(1.3). A microlocal parametrix will be constructed as the composition of a microlocal parametrix for the Dirichlet problem and a microlocal parametrix for a system of pseudo differential operators on the boundary in $\S 5$.

The author wishes to thank Professor M. Matsumura for his helpful discussions and his encouragement.

$\dagger$ It is easy to see that for any $f \in C^{\infty}\left([0, \varepsilon) ; \mathscr{D}^{\prime}(U)\right)$ there is $F \in C^{\infty}\left((-\varepsilon, \varepsilon) ; \mathscr{D}^{\prime}(U)\right)$ such that $f=\left.F\right|_{x_{0} \geq_{0}}$. 


\section{§2. Formal Construction}

In this section we first determine the boundary values of the phase functions and solve the eiconal equations to determine the phase functions. Then we discuss the transport equation on the boundary and the transport equations.

There exist a conic neighborhood $\Gamma_{0}$ of $\left(x^{0^{\prime}}, \xi^{0^{\prime}}\right)$ in $T^{*} \mathbb{R}^{n} \backslash 0$ and $\tilde{\phi}\left(x^{\prime}, \xi^{\prime}\right) \in C^{\infty}\left(\Gamma_{0}\right)$ such that $\tilde{\phi}\left(x^{\prime}, \xi^{\prime}\right)$ satisfies the equations

$$
\begin{aligned}
& \partial_{1} \tilde{\psi}\left(x^{\prime}, \xi^{\prime}\right)-\xi_{1}\left(x^{\prime}, \nabla_{x^{\prime \prime}} \tilde{\psi}\left(x^{\prime}, \xi^{\prime}\right)\right)=\xi_{1}-\xi_{1}\left(x^{0^{\prime}}, \xi^{\prime \prime}\right), \\
& \tilde{\psi}\left(x_{1}^{0}, x^{\prime \prime}, \xi^{\prime}\right)=x^{\prime \prime} \cdot \xi^{\prime \prime},
\end{aligned}
$$

where $\partial_{j}=\partial_{x_{j}}=\partial / \partial x_{j}$ and $\nabla_{x^{\prime \prime}} f=\left(\partial_{2} f, \cdots, \partial_{n} f\right)$. Moreover $\tilde{\psi}\left(x^{\prime}, \xi^{\prime}\right)$ is homogeneous of degree 1 in $\xi^{\prime}$. Let $\chi\left(x^{\prime}, y^{\prime}, \xi^{\prime}\right)$ be a $C^{\infty}$ function in $\mathbb{R}^{3 n}$ such that $\chi=1$ in $\dot{\Gamma}_{2} \cap\left\{\left|\xi^{\prime}\right| \geqq 1\right\}$ and supp $\chi \subset \dot{\Gamma}_{1}$, where $\Gamma_{1}\left(\subset \subset^{\dagger} \Gamma_{0}\right)$ and $\Gamma_{2}$ are conic neighborhood of $\left(x^{0^{\prime}}, \xi^{0^{\prime}}\right)$ in $T^{*} \mathbb{R}^{n} \backslash 0$ and

$$
\dot{\Gamma}=\left\{\left(x^{\prime}, y^{\prime}, \xi^{\prime}\right) ;\left(x^{\prime}, \xi^{\prime}\right) \in \Gamma \text { and }\left(y^{\prime}, \xi^{\prime}\right) \in \Gamma\right\} .
$$

Since $\left(\partial^{2} / \partial x_{j} \partial \xi_{k} \tilde{\psi}\left(x^{0^{\prime}}, \xi^{0^{\prime}}\right)\right)=I$, it follows that the operator $A$ :

$$
\begin{aligned}
\mathscr{D}^{\prime}\left(\mathbb{R}^{n}\right) \ni g\left(x^{\prime}\right) \rightarrow(A g)\left(x^{\prime}\right)= & \int \exp \left[i\left(\tilde{\psi}\left(x^{\prime}, \xi^{\prime}\right)-\tilde{\psi}\left(y^{\prime}, \xi^{\prime}\right)\right)\right] \\
& \times \chi\left(x^{\prime}, y^{\prime}, \xi^{\prime}\right) g\left(y^{\prime}\right) d y^{\prime} d \xi^{\prime} \in \mathscr{D}^{\prime}\left(\mathbb{R}^{n}\right)
\end{aligned}
$$

is a properly supported pseudo-dieffrential operator, if necessary, shrinking $\Gamma_{1}$, where $d \xi^{\prime}=(2 \pi)^{-n} d \xi^{\prime} . A$ is elliptic in a conic neighborhood of $\left(x^{\prime \prime}, \xi^{\prime \prime}\right)$. Thus there is a microlocal parametrix (pseudodifferential operator) $B$ of $A$ at $\left(x^{0^{\prime}}, \xi^{0^{\prime}}\right)$, i.e., there exists a conic neighborhood $\Gamma$ of $\left(x^{0^{\prime}}, \xi^{0^{\prime}}\right)$ such that $A B g-g \in C^{\infty}\left(\mathbb{R}^{n}\right)$ if $W F(g) \subset \Gamma$.

Let us formally construct a microlocal parametrix for the problem $(1.1)^{\prime}-(1.3)^{\prime}$ with $k=1$ at $\left(x^{0}, \xi^{\prime \prime}\right)$ in the form

$$
\begin{aligned}
E_{1}(g)= & \sum_{j=1}^{l_{1}} \int \exp \left[i \phi_{j}\left(x, y^{\prime}, \xi^{\prime}\right)\right] a_{j}\left(x, y^{\prime}, \xi^{\prime}\right)(B g)\left(y^{\prime}\right) d y^{\prime} d \xi^{\prime} \\
& +\int \exp \left[i\left(\tilde{\psi}\left(x^{\prime}, \xi^{\prime}\right)-\tilde{\psi}\left(y^{\prime}, \xi^{\prime}\right)\right)\right] a\left(x, y^{\prime}, \xi^{\prime}\right)(B g)\left(y^{\prime}\right) d y^{\prime} d \xi^{\prime}
\end{aligned}
$$

$+\Gamma_{1} \subset \subset \Gamma_{0}$ means that the closure of $\Gamma_{1}$ is included in the interior of $\Gamma_{0}$. 


$$
a\left(x, y^{\prime}, \xi^{\prime}\right)=\sum_{j=1}^{l-l_{1}} \frac{1}{2 \pi i} \int_{c \xi^{\prime}} c_{j}\left(x, y^{\prime}, \xi\right) \xi_{0}^{j-1} \exp \left[i x_{0} \xi_{0}\right] d \xi_{0} .
$$

Then we have

$$
\begin{aligned}
& P E_{1}(g)=\sum_{j=1}^{l_{1}} \int \exp \left[i \phi_{j}\left(x, y^{\prime}, \xi^{\prime}\right)\right]\left\{p\left(x, \nabla_{x} \phi_{j}\right)\right. \\
& \left.\quad+\sum_{|\alpha|=1} p^{(\alpha)}\left(x, \nabla_{x} \phi_{j}\right) D^{\alpha}+s\left(\phi_{j} ; x\right)+q\left(\phi_{j} ; x, D\right)\right\} a_{j}\left(x, y^{\prime}, \xi^{\prime}\right) \\
& \left.\quad \times(B g)\left(y^{\prime}\right) d y^{\prime} \tilde{d} \xi^{\prime}+\int \exp \left[i \tilde{\psi}\left(x^{\prime}, \xi^{\prime}\right)-\tilde{\psi}\left(y^{\prime}, \xi^{\prime}\right)\right)\right] \\
& \quad \times\left[\sum _ { j = 1 } ^ { l - l _ { 1 } } \frac { 1 } { 2 \pi i } \int _ { c _ { \xi } } \left\{p\left(x, \nabla_{x} \psi_{0}\right)+\sum_{|\alpha|=1} p^{(\alpha)}\left(x, \nabla_{x} \psi_{0}\right) D^{\alpha}+s\left(\phi_{0} ; x\right)\right.\right. \\
& \left.\left.\quad+q\left(\phi_{0} ; x, D\right)\right\} c_{j}\left(x, y^{\prime}, \xi\right) \xi_{0}^{j-1} \exp \left[i x_{0} \xi_{0}\right] d \xi_{0}\right] d y^{\prime} d \xi^{\prime},
\end{aligned}
$$

where $\quad p^{1}(x, \xi)=P(x, \xi)-p(x, \xi), \quad p^{(\alpha)}(x, \zeta)=\partial_{\zeta}^{\alpha} p(x, \zeta), \quad \phi_{0}(x, \xi)$ $=\tilde{\phi}\left(x^{\prime}, \xi^{\prime}\right)+x_{0} \xi_{0}$,

$$
\begin{aligned}
& s(\phi ; x)=p^{1}\left(x, \nabla_{x} \phi\right)+\sum_{|\alpha|=2} \frac{1}{\alpha !} p^{(\alpha)}\left(x, \nabla_{x} \phi\right)\left(i D^{\alpha} \phi\right), \\
& q(\phi ; x, D) f(x)=\left\{\sum_{|\alpha|=1} p^{1(\alpha)}\left(x, \nabla_{x} \phi\right) D^{\alpha}+\sum_{|\alpha|=2} \frac{1}{\alpha !} p^{1(\alpha)}\left(x, \nabla_{x} \phi\right)\right. \\
& \left.\quad \times\left(i D^{\alpha} \phi\right)+\sum_{|\alpha|=2} \frac{1}{\alpha !} P^{(\alpha)}\left(x, \nabla_{x} \phi\right) D^{\alpha}\right\} f(x) \\
& \quad+\sum_{|\alpha| \geq 3} \frac{1}{\alpha !} P^{(\alpha)}\left(x, \nabla_{x} \phi\right) \cdot D_{z}\{f(z) \exp [i h(\phi ; x, z)]\}_{z=x}, \\
& h(\phi ; x, z)=\phi(z)-\phi(x)-(z-x) \cdot \nabla_{x} \phi(x) .
\end{aligned}
$$

Thus $\dot{\phi}_{j}\left(x, y^{\prime}, \xi^{\prime}\right), 1 \leqq j \leqq l_{1}$, are determined by the eiconal equations

$$
\partial_{0} \phi_{j}\left(x, \xi^{\prime}\right)=\lambda_{j}^{+}\left(x, \nabla_{x^{\prime}} \phi_{j}\right), \phi_{j}\left(0, x^{\prime}, \xi^{\prime}\right)=\tilde{\phi}\left(x^{\prime}, \xi^{\prime}\right),
$$

where $\phi_{j}\left(x, y^{\prime}, \xi^{\prime}\right)=\phi_{j}\left(x, \xi^{\prime}\right)-\phi_{j}\left(0, y^{\prime}, \xi^{\prime}\right)$. We easily see that $\phi_{j}\left(x, y^{\prime}, \xi^{\prime}\right) \in C^{\infty}\left([0, \varepsilon) \times \dot{\Gamma}_{0}\right)$ for some $\varepsilon>0$, if necessary, shrinking $\Gamma_{0}$. If $a_{j}\left(x, y^{\prime}, \xi^{\prime}\right), 1 \leqq j \leqq l_{1}$, can be written as asymptotic sums

$$
a_{j}\left(x, y^{\prime}, \xi^{\prime}\right) \sim \sum_{\nu=0}^{\infty} a_{j}^{\nu}\left(x, y^{\prime}, \xi^{\prime}\right)
$$

in a certain sense, we obtain the transport equations

$$
\begin{aligned}
& \left\{\sum_{|\alpha|=1} p^{(\alpha)}\left(x, \nabla_{x} \phi_{j}\right) D^{\alpha}+s\left(\phi_{j} ; x\right)\right\} a_{j}^{\nu}\left(x, y^{\prime}, \xi^{\prime}\right) \\
& \quad+q\left(\phi_{j} ; x, D\right) a_{j}^{\nu-1}\left(x, y^{\prime}, \xi^{\prime}\right)=0 \\
& a_{j}^{-1}\left(x, y^{\prime}, \xi^{\prime}\right) \equiv 0, \quad 1 \leqq j \leqq l_{1}, \quad \nu=0,1,2, \ldots
\end{aligned}
$$

(2. 6) is an ordinary differential equation for $a_{j}^{y}$ along rays 
corresponding to (2.4). Thus we can solve the transport equations (2.6) when the boundary values of $a_{j}^{y}$ are given. Put

$$
a_{j}^{\nu}\left(0, x^{\prime}, y^{\prime}, \xi^{\prime}\right)=\tilde{a}_{j}^{\nu}\left(x^{\prime}, y^{\prime}, \xi^{\prime}\right) \in C^{\infty}\left(\dot{\Gamma}_{0}\right), 1 \leqq j \leqq l_{1}, \nu=0,1, \cdots .
$$

Then we can assume without loss of generality that the solutions $a_{j}^{\nu}\left(x, y^{\prime}, \xi^{\prime}\right)$ belong to $C^{\infty}\left([0, \varepsilon) \times \dot{\Gamma}_{0}\right)$. Moreover from $(2.6)$ we have

$$
\begin{aligned}
& D_{0} a_{j}^{\nu}\left(0, x^{\prime}, y^{\prime}, \xi^{\prime}\right)=\sum_{k=1}^{n} \partial \lambda_{j}^{+} / \partial \zeta_{k}\left(0, x^{\prime}, \nabla_{x^{\prime}} \tilde{\psi}\right) D_{k} \tilde{a}_{j}^{\nu}\left(x^{\prime}, y^{\prime}, \xi^{\prime}\right) \\
& \quad+s_{j}\left(x^{\prime}, \xi^{\prime}\right) \tilde{a}_{j}^{\nu}\left(x^{\prime}, y^{\prime}, \xi^{\prime}\right)+q_{j}\left(x^{\prime}, \xi^{\prime}, D\right) a_{j}^{\nu-1}\left(0, x^{\prime}, y^{\prime}, \xi^{\prime}\right),
\end{aligned}
$$

where $D_{0}^{k} f\left(0, x^{\prime}\right)=\left.D_{0}^{k} f(x)\right|_{x_{0}=0}, \partial \lambda_{j}^{+} / \partial \zeta_{k}\left(0, x^{\prime}, \nabla_{x^{\prime}} \tilde{\psi}\right)=\partial \lambda_{j}^{+} /\left.\partial \zeta_{k}\left(0, x^{\prime}, \zeta^{\prime}\right)\right|_{\zeta=\nabla s^{\prime} \psi}$, $p^{(j)}(x, \zeta)=\partial p / \partial \zeta_{j}(x, \zeta)$,

$$
\begin{aligned}
& s_{j}\left(x^{\prime}, \xi^{\prime}\right)=-p^{(0)}\left(0, x^{\prime}, \lambda_{j}^{+}\left(0, x^{\prime}, \nabla_{x^{\prime}} \tilde{\psi}\right), \nabla_{x^{\prime}} \tilde{\psi}\right)^{-1} s\left(\phi_{j} ; 0, x^{\prime}\right), \\
& q_{j}\left(x^{\prime}, \xi^{\prime}, D\right)=-p^{(0)}\left(0, x^{\prime}, \lambda_{j}^{+}, \nabla_{x^{\prime}} \tilde{\psi}\right)^{-1} q\left(\phi_{j} ; 0, x^{\prime}, D\right) .
\end{aligned}
$$

We represent $c_{j}\left(x, y^{\prime}, \xi\right)$ as asymptotic sums

$$
c_{j}\left(x, y^{\prime}, \xi\right) \sim \sum_{\nu, \mu=0}^{\infty} c_{j}^{\nu \mu}\left(x, y^{\prime}, \xi\right) .
$$

From (2.3) we put

$$
\begin{array}{cc}
\text { (2. 10) } & c_{j}^{\nu 0}\left(x, y^{\prime}, \xi\right)=\tilde{c}_{j}^{\nu}\left(x^{\prime}, y^{\prime}, \xi^{\prime}\right) \rho\left(x_{0}\right) p\left(x, \nabla_{x} \psi_{0}\right)^{-1}, \\
(2.11) & c_{j}^{\nu \mu+1}\left(x, y^{\prime}, \xi\right)=-\left[\left\{\sum_{|\alpha|=1} p^{(\alpha)}\left(x, \nabla_{x} \psi_{0}\right) D^{\alpha}+s\left(\psi_{0} ; x\right)\right\} c_{j}^{\nu \mu}\left(x, y^{\prime}, \xi\right)\right. \\
+ & \left.q\left(\psi_{0} ; x, D\right) c_{j}^{\nu-1 \mu}\left(x, y^{\prime}, \xi\right)\right] p\left(x, \nabla_{x} \psi_{0}\right)^{-1}, \\
c_{j}^{-1 \mu} \equiv 0, \quad 1 \leqq j \leqq l-l_{1}, \quad \nu, \mu=0,1,2, \cdots,
\end{array}
$$

where $\rho\left(x_{0}\right) \in C_{(0)}^{\infty}([0, \varepsilon))^{\dagger}, \rho\left(x_{0}\right)=1$ in a neighborhood of $x_{0}=0$ and the $\tilde{c}_{j}^{\nu}\left(x^{\prime}, y^{\prime}, \xi^{\prime}\right) \in C^{\infty}(\dot{\Gamma})$ will be determined by the transport equations on the boundary. Then we have $P E_{1}(g) \sim 0$ in some sense.

Next let us determine the $\tilde{a}_{j}^{\nu}\left(x^{\prime}, y^{\prime}, \xi^{\prime}\right)$ and the $\tilde{c}_{j}^{\nu}\left(x^{\prime}, y^{\prime}, \xi^{\prime}\right)$. We obtain formally

$$
\begin{aligned}
& \left.B_{k} E_{1}(g)\right|_{x_{0}=0}=\sum_{j=1}^{l_{1}} \int \exp \left[i\left(\tilde{\psi}\left(x^{\prime}, \xi^{\prime}\right)-\tilde{\psi}\left(y^{\prime}, \xi^{\prime}\right)\right)\right] \\
& \quad \times\left\{b_{k}\left(x^{\prime}, \lambda_{j}^{+}\left(0, x^{\prime}, \nabla_{x^{\prime}} \tilde{\psi}\left(x^{\prime}, \xi^{\prime}\right)\right), \nabla_{x^{\prime}} \tilde{\psi}\right)\right. \\
& \quad+\sum_{|\alpha|=1} b_{k}^{(\alpha)}\left(x^{\prime}, \lambda_{j}^{+}, \nabla_{x^{\prime}} \tilde{\psi}\right) D^{\alpha}+h_{k}\left(\psi_{j} ; x^{\prime}\right) \\
& \left.\quad+t_{k}\left(\phi_{j} ; x^{\prime}, D\right)\right\} a_{j}\left(0, x^{\prime}, y^{\prime}, \xi^{\prime}\right)(B g)\left(y^{\prime}\right) d y^{\prime} d \xi^{\prime} \\
& \quad+\int \exp \left[i\left(\tilde{\psi}\left(x^{\prime}, \xi^{\prime}\right)-\tilde{\psi}\left(y^{\prime}, \xi^{\prime}\right)\right)\right]\left[\sum _ { j = 1 } ^ { l - l 1 } \frac { 1 } { 2 \pi i } \int _ { c _ { \xi ^ { \prime } } } \left\{b_{k}\left(x^{\prime}, \nabla_{x} \psi_{0}\right)\right.\right.
\end{aligned}
$$

$\dagger C_{(0)}^{\infty}([0, \varepsilon))=\left\{f\left(x_{0}\right) \in C^{\infty}([0, \varepsilon))\right.$; supp $f$ is compact $\}$. 


$$
\begin{aligned}
& \left.+\sum_{|\alpha|=1} b_{k}^{(\alpha)}\left(x^{\prime}, \nabla_{x} \phi_{0}\right) D^{\alpha}+h_{k}\left(\psi_{0} ; x^{\prime}\right)+t_{k}\left(\psi_{0} ; x^{\prime}, D\right)\right\} \\
& \left.\times c_{j}\left(0, x^{\prime}, y^{\prime}, \xi\right) \xi_{0}^{j-1} d \xi_{0}\right](B g)\left(y^{\prime}\right) d y^{\prime} d \xi^{\prime},
\end{aligned}
$$

where $b_{k}^{1}\left(x^{\prime}, \xi\right)=B_{k}\left(x^{\prime}, \xi\right)-b_{k}\left(x^{\prime}, \xi\right)$,

$$
\begin{aligned}
& h_{k}\left(\phi ; x^{\prime}\right)= b_{k}^{1}\left(x^{\prime}, \nabla_{x} \phi\left(0, x^{\prime}\right)\right)+\sum_{|\alpha|=2} \frac{1}{\alpha !} b_{k}^{(\alpha)}\left(x^{\prime}, \nabla_{x} \phi\left(0, x^{\prime}\right)\right) \\
& \times\left(i D^{\alpha} \phi\left(0, x^{\prime}\right)\right), \\
& t_{k}\left(\phi ; x^{\prime}, D\right) f\left(0, x^{\prime}\right)=\left\{\sum_{|\alpha|=1} b_{k}^{1(\alpha)}\left(x^{\prime}, \nabla_{x} \phi\left(0, x^{\prime}\right)\right) D^{\alpha}\right. \\
&\left.\quad+\sum_{|\alpha|=2} \frac{1}{\alpha !} b_{k}^{1(\alpha)}\left(x^{\prime}, \nabla_{x} \phi\right)\left(i D^{\alpha} \phi\left(0, x^{\prime}\right)\right)+\sum_{|\alpha|=2} \frac{1}{\alpha !} B_{k}^{(\alpha)}\left(x^{\prime}, \nabla_{x} \phi\right) D^{\alpha}\right\} \\
& \quad \times f\left(0, x^{\prime}\right)+\sum_{|\alpha| \geq 3} \frac{1}{\alpha !} B_{k}^{(\alpha)}\left(x^{\prime}, \nabla_{x} \phi\right) D_{z}^{\alpha}\left\{f(z) e^{i h(\phi x, z)}\right\}_{z=x=\left(0, x^{\prime}\right)} .
\end{aligned}
$$

Thus we have the transport equations on the boundary

$$
\begin{aligned}
& \sum_{j=1}^{l_{1}} b_{k}\left(x^{\prime}, \lambda_{j}^{+}\left(0, x^{\prime}, \nabla_{x^{\prime}} \tilde{\psi}\left(x^{\prime}, \xi^{\prime}\right)\right), \nabla_{x^{\prime}} \tilde{\psi}\right) \tilde{a}_{j}^{\nu}\left(x^{\prime}, y^{\prime}, \xi^{\prime}\right) \\
& +\sum_{j=1}^{l-l_{1}}\left(\frac{1}{2 \pi i} \int_{c \xi^{\prime}} \frac{b_{k}\left(x^{\prime}, \xi_{0}, \nabla_{x^{\prime}} \tilde{\phi}\right) \xi_{0}^{j-1}}{\left(0, x^{\prime}, \xi_{0}, \nabla_{x^{\prime}} \tilde{\psi}\right)} d \xi_{0}\right) \tilde{c}_{j}^{\nu}\left(x^{\prime}, y^{\prime}, \xi^{\prime}\right) \\
& +\sum_{j=1}^{l_{1}} \sum_{s=1}^{n}\left(b_{k}^{(s)}\left(x^{\prime}, \lambda_{j}^{+}, \nabla_{x^{\prime}} \tilde{\psi}\right)+b_{k}^{(0)}\left(x^{\prime}, \lambda_{j}^{+}, \nabla_{x^{\prime}} \tilde{\psi}\right) \frac{\partial \lambda_{j}^{+}}{\partial \zeta_{s}}\left(0, x^{\prime}, \nabla_{x^{\prime}} \tilde{\psi}\right)\right) \\
& \times D_{s} \tilde{a}_{j}^{\nu}\left(x^{\prime}, y^{\prime}, \xi^{\prime}\right)+\sum_{j=1}^{l-l_{1}} \sum_{s=1}^{n}\left\{\frac { 1 } { 2 \pi i } \int _ { c _ { \xi } ^ { \prime } } \left[b_{k}^{(s)}\left(x^{\prime}, \xi_{0}, \nabla_{x^{\prime}} \tilde{\psi}\right)\right.\right. \\
& \left.\left.\times p\left(0, x^{\prime}, \xi_{0}, \nabla_{x^{\prime}} \tilde{\psi}\right)-b_{k} p^{(s)}\right] \xi_{0}^{j-1} p^{-2} d \xi_{0}\right\} D_{s} \tilde{c}_{j}^{\nu}\left(x^{\prime}, y^{\prime}, \xi^{\prime}\right) \\
& +\sum_{j=1}^{l_{1}} S_{k j}\left(x^{\prime}, \xi^{\prime}\right) \tilde{a}_{j}^{\nu}\left(x^{\prime}, y^{\prime}, \xi^{\prime}\right)+\sum_{j=1}^{l-l_{1}} S_{k j}^{0}\left(x^{\prime}, \xi^{\prime}\right) \tilde{c}_{j}^{\nu}\left(x^{\prime}, y^{\prime}, \xi^{\prime}\right) \\
& =\delta_{\nu 0} \delta_{k 1} \chi\left(x^{\prime}, y^{\prime}, \xi^{\prime}\right)+f_{k}^{\nu}\left(x^{\prime}, y^{\prime}, \xi^{\prime}\right), 1 \leqq k \leqq l, \nu=0,1,2, \cdots,
\end{aligned}
$$

where

$$
\begin{aligned}
& S_{k j}\left(x^{\prime}, \xi^{\prime}\right)=h_{k}\left(\psi_{j} ; x^{\prime}\right)+b_{k}^{(0)}\left(x^{\prime}, \lambda_{j}^{+}, \nabla_{x^{\prime}} \tilde{\psi}\right) s_{j}\left(x^{\prime}, \xi^{\prime}\right), \\
& S_{k j}^{0}\left(x^{\prime}, \xi^{\prime}\right)=\sum_{j=1}^{l-l_{1}} \frac{1}{2 \pi i} \int_{c_{\xi^{\prime}}}\left\{h_{k}\left(\psi_{0} ; x^{\prime}\right)\right. \\
& -\sum_{|\alpha|=1} b_{k}^{(\alpha)}\left(x^{\prime}, \xi_{0}, \nabla_{x^{\prime}} \tilde{\psi}\right) \frac{D^{\alpha} p\left(0, x^{\prime}, \xi_{0}, \nabla_{x^{\prime}} \tilde{\psi}\right)}{p\left(0, x^{\prime}, \xi_{0}, \nabla_{x^{\prime}} \tilde{\psi}\right)}-b_{k} \cdot h \cdot p^{-1} \\
& \left.+\sum_{|\alpha|=1} b_{k} \cdot\left(D^{\alpha} p\right) \cdot p^{(\alpha)} \cdot p^{-2}\right\} \xi_{0}^{j-1} p^{-1} d \xi_{0},
\end{aligned}
$$

(2. 14) $f_{k}^{\nu}\left(x^{\prime}, y^{\prime}, \xi^{\prime}\right) \sim-\sum_{j=1}^{l_{1}}\left\{b_{k}^{(0)}\left(x^{\prime}, \lambda_{j}^{+}\left(0, x^{\prime}, \nabla_{x^{\prime}} \tilde{\psi}\right), \nabla_{x^{\prime}} \tilde{\psi}\right) q_{j}\left(x^{\prime}, \xi^{\prime}, D\right)\right.$

$\left.+t_{k}\left(\phi_{0} ; x^{\prime}, D\right)\right\} a_{j}^{\nu-1}\left(0, x^{\prime}, y^{\prime}, \xi^{\prime}\right)$

$-\sum_{j=1}^{l-l_{1} 1} \frac{1}{2 \pi i} \int_{c_{\xi^{\prime}}}\left[-\frac{b_{k}\left(x^{\prime}, \xi_{0}, \nabla_{x^{\prime}} \tilde{\psi}\right)}{p\left(0, x^{\prime}, \xi_{0}, \nabla_{x^{\prime}} \tilde{\psi}\right)} q\left(\psi_{0} ; 0, x^{\prime}, D\right) c_{j}^{\nu-10}\left(0, x^{\prime}, y^{\prime}, \xi\right)\right.$

$+\sum_{\mu=0}^{\infty} t_{k}\left(\psi_{0} ; x^{\prime}, D\right) c_{j}^{\nu-1 \mu}\left(0, x^{\prime}, y^{\prime}, \xi\right)+\sum_{\mu=1}^{\infty}\left\{\sum_{|\alpha|=1} b_{k}^{(\alpha)} D^{\alpha}\right.$ 


$$
\begin{gathered}
\left.\left.+h_{k}\left(\phi_{0} ; x^{\prime}\right)\right\} c_{j}^{\nu-1 \mu}\left(0, x^{\prime}, y^{\prime}, \xi\right)+\sum_{\mu=2}^{\infty} b_{k} c_{J}^{\nu-1 \mu}\left(0, x^{\prime}, y^{\prime}, \xi\right)\right] \xi_{0}^{j-1} d \xi_{0} \\
\nu=1,2, \cdots,
\end{gathered}
$$

$$
f_{k}^{0}\left(x^{\prime}, y^{\prime}, \xi^{\prime}\right) \equiv 0 \text {. }
$$

In fact (2.13) follows from (2.8) and (2. 10)-(2.12). Putting

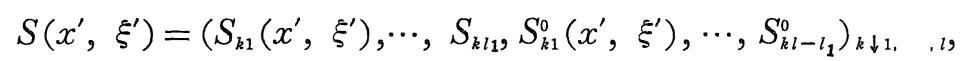

$$
\begin{aligned}
& \text { (2. 16) } a^{\nu}\left(x^{\prime}, y^{\prime}, \xi^{\prime}\right)={ }^{t}\left(\tilde{a}_{1}^{\nu}\left(x^{\prime}, y^{\prime}, \xi^{\prime}\right), \cdots, \tilde{a}_{l_{1}}^{\nu}, \tilde{c}_{1}^{\nu}\left(x^{\prime}, y^{\prime}, \xi^{\prime}\right),\right. \\
& \left.\cdots, \tilde{c}_{l-l_{1}}^{\nu}\right), \\
& \text { (2. 17) } F^{\nu}\left(x^{\prime}, y^{\prime}, \xi^{\prime}\right)={ }^{t}\left(\delta_{\nu 0} \chi\left(x^{\prime}, y^{\prime}, \xi^{\prime}\right)+f_{1}^{\nu}, f_{2}^{\nu}, \cdots, f_{l}^{\nu}\right),
\end{aligned}
$$

we can rewrite (2.13) in the form

$$
\begin{aligned}
& L\left(x^{\prime}, \nabla_{x^{\prime}} \tilde{\psi}\left(x^{\prime}, \xi^{\prime}\right)\right) a^{\nu}\left(x^{\prime}, y^{\prime}, \xi^{\prime}\right)+\sum_{j=1}^{n} \partial L / \partial \zeta_{j} D_{j} a^{\nu}\left(x^{\prime}, y^{\prime}, \xi^{\prime}\right) \\
& +S\left(x^{\prime}, \xi^{\prime}\right) a^{\nu}\left(x^{\prime}, y^{\prime}, \xi^{\prime}\right)=\xi^{\nu}\left(x^{\prime}, y^{\prime}, \xi^{\prime}\right), \nu=0,1,2, \cdots,
\end{aligned}
$$

where $\partial L / \partial \zeta_{j}=\partial L /\left.\partial \zeta_{j}\left(x^{\prime}, \zeta^{\prime}\right)\right|_{\zeta^{\prime}=\nabla_{x^{\prime}} \dot{\varphi}}$. Put

$$
\boldsymbol{a}^{\nu}\left(x^{\prime}, y^{\prime}, \xi^{\prime}\right)=\left(V\left(x^{\prime}, \nabla_{x^{\prime}} \tilde{\psi}\right)+\sum_{j=1}^{n} \partial V / \partial \zeta_{j} D_{j}\right) \mathscr{v}^{\nu}\left(x^{\prime}, y^{\prime}, \xi^{\prime}\right) .
$$

Multiplying (2.18) by $U\left(x^{\prime}, \nabla_{x^{\prime}} \tilde{\psi}\right)+\sum_{k=1}^{n} \partial U / \partial \zeta_{k} D_{k}$ and using (A.3) and (2.1) we have

$$
\begin{aligned}
& \left(\xi_{1}-\xi_{1}\left(x^{0^{\prime}}, \xi^{\prime \prime}\right)\right) v_{k}^{\nu}\left(x^{\prime}, y^{\prime}, \xi^{\prime}\right)+\left\{D_{1}-\sum_{j=2}^{n} \partial \xi_{1} / \partial \zeta_{j}\left(x^{\prime}, \nabla_{x^{\prime \prime}} \tilde{\psi}\right) D_{\jmath}\right\} \\
& \times v_{j}^{\nu}\left(x^{\prime}, y^{\prime}, \xi^{\prime}\right)=G_{k}^{\nu}\left(x^{\prime}, y^{\prime}, \xi^{\prime}\right), \quad 1 \leqq k \leqq \theta^{\prime}, \\
& L_{e}\left(x^{\prime}, \nabla_{x^{\prime}} \tilde{\psi}\left(x^{\prime}, \xi^{\prime}\right)\right) \boldsymbol{v}_{e}^{\nu}\left(x^{\prime}, y^{\prime}, \xi^{\prime}\right)+Q_{e}^{1}\left(x^{\prime}, \xi^{\prime}, D^{\prime}\right) V_{\theta^{\prime}}^{\nu}\left(x^{\prime}, y^{\prime}, \xi^{\prime}\right) \\
& \quad+Q_{e}^{2}\left(x^{\prime}, \xi^{\prime}, D^{\prime}\right) \boldsymbol{v}_{e}^{\nu}\left(x^{\prime}, y^{\prime}, \xi^{\prime}\right)=G_{e}^{\nu}\left(x^{\prime}, y^{\prime}, \xi^{\prime}\right),
\end{aligned}
$$

where $\boldsymbol{v}^{\nu}={ }^{t}\left(v_{1}^{\nu}, \cdots, v_{l}^{\nu}\right), \boldsymbol{v}_{\theta^{\prime}}^{\nu}={ }^{t}\left(v_{1}^{\nu}, \cdots, v_{\theta^{\prime}}^{\nu}\right), \boldsymbol{v}_{e}^{\nu}={ }^{t}\left(v_{\theta^{\prime}+1}^{\nu}, \cdots, v_{l}^{\nu}\right)$,

$$
\begin{aligned}
& T\left(x^{\prime}, \xi^{\prime}\right)\left(\equiv\left(T_{i j}\left(x^{\prime}, \xi^{\prime}\right)\right)\right)=\sum_{k=1}^{n}\left[\partial U / \partial \zeta_{j}\left(x^{\prime}, \nabla_{x^{\prime}} \tilde{\psi}\left(x^{\prime}, \xi^{\prime}\right)\right)\right. \\
& \left.\quad \times\left\{D_{j}\left(L\left(x^{\prime}, \nabla_{x^{\prime}} \tilde{\psi}\right) V\left(x^{\prime}, \nabla_{x^{\prime}} \tilde{\psi}\right)\right)\right\}+U \partial L / \partial \zeta_{j}\left(D_{j} V\right)\right] \\
& \quad+U S\left(x^{\prime}, \xi^{\prime}\right) V \\
& \quad W\left(x^{\prime}, \xi^{\prime}, D^{\prime}\right) \boldsymbol{f}\left(x^{\prime}\right)\left(\equiv\left(W_{i j}\left(x^{\prime}, \xi^{\prime}, D^{\prime}\right)\right) \boldsymbol{f}\left(x^{\prime}\right)\right) \\
& \quad=\sum_{j, k=1}^{n}\left\{\partial U / \partial \zeta_{j} D_{j}\left(L \partial V / \partial \zeta_{k} D_{k} f\left(x^{\prime}\right)\right)\right. \\
& \left.\quad+U \partial L / \partial \zeta_{j} D_{j}\left(\partial V / \partial \zeta_{k} D_{k} f\left(x^{\prime}\right)\right)\right\} \\
& \quad+\sum_{i, j, k=1}^{n} \partial U / \partial \zeta_{i} D_{i}\left[\partial L / \partial \zeta_{j} D_{j}\left\{\left(V+\partial V / \partial \zeta_{k} D_{k}\right) f\left(x^{\prime}\right)\right\}\right] \\
& \quad+\sum_{j, k=1}^{n} U S \partial V / \partial \zeta_{j} D_{j} f\left(x^{\prime}\right)+\sum_{j=1}^{n} \partial U / \partial \zeta_{j} D_{j}\{S(V \\
& \left.\left.\quad+\sum_{k=1}^{n} \partial V / \partial \zeta_{k} D_{k}\right) \boldsymbol{f}\left(x^{\prime}\right)\right\}, \\
& \boldsymbol{G}^{\nu}\left(x^{\prime}, y^{\prime}, \xi^{\prime}\right)={ }^{t}\left(G_{1}^{\nu}\left(x^{\prime}, y^{\prime}, \xi^{\prime}\right), \cdots, G_{l}^{\nu}\left(x^{\prime}, y^{\prime}, \xi^{\prime}\right)\right)
\end{aligned}
$$




$$
\begin{aligned}
& =\left(U+\sum_{j=1}^{n} \partial U / \partial \zeta_{j} D_{j}\right) F^{\nu}\left(x^{\prime}, y^{\prime}, \xi^{\prime}\right), \\
& T_{e}^{1}=\left(T_{j k}\right)_{\substack{j \downarrow \theta^{\prime}+1, \ldots, l \\
k \rightarrow 1, \ldots, \theta^{\prime}}} T_{e}^{2}=\left(T_{j k}\right)_{j, k=\theta^{\prime}+1, \ldots, l}, \\
& Q_{e}^{1}\left(x^{\prime}, \xi^{\prime}, D^{\prime}\right)=T_{e}^{1}\left(x^{\prime}, \xi^{\prime}\right)+W_{e}^{1}\left(x^{\prime}, \xi^{\prime}, D^{\prime}\right), \\
& Q_{e}^{2}\left(x^{\prime}, \xi^{\prime}, D^{\prime}\right)=\sum_{j=1}^{n} \partial L_{e} / \partial \zeta_{j}\left(x^{\prime}, \nabla_{x^{\prime}} \tilde{\psi}\right) D_{j}+T_{e}^{2}+W_{e \bullet}^{2}
\end{aligned}
$$

Representing $\boldsymbol{v}^{\nu}\left(x^{\prime}, y^{\prime}, \xi^{\prime}\right)$ as asymptotic sums

$$
\boldsymbol{v}^{\nu}\left(x^{\prime}, y^{\prime}, \xi^{\prime}\right) \sim \sum_{\mu=0}^{\infty} \boldsymbol{v}^{\nu \mu}\left(x^{\prime}, y^{\prime}, \xi^{\prime}\right), \nu=0,1,2, \cdots,
$$

we have

$$
\begin{aligned}
& \left(\xi_{1}-\xi_{1}\left(x^{0^{\prime}}, \xi^{\prime \prime}\right)\right) v_{k}^{\nu \mu}\left(x^{\prime}, y^{\prime}, \xi^{\prime}\right)+\left(D_{1}-\sum_{j=2}^{n} \partial \xi_{1} / \partial \zeta_{j} D_{j}\right) \\
& \quad \times v_{k}^{\nu \mu}\left(x^{\prime}, y^{\prime}, \xi^{\prime}\right)+\sum_{j=1}^{\theta^{\prime}} T_{k j}\left(x^{\prime}, \xi^{\prime}\right) v_{j}^{\nu \mu}\left(x^{\prime}, y^{\prime}, \xi^{\prime}\right) \\
& \quad=\delta_{\mu 0} G_{k}^{\nu}\left(x^{\prime}, y^{\prime}, \xi^{\prime}\right)-\sum_{j=\theta^{\prime}+1}^{l} T_{k j} v_{j}^{\nu \mu-1}\left(x^{\prime}, y^{\prime}, \xi^{\prime}\right) \\
& \quad-\sum_{j=1}^{l} W_{k j}\left(x, \xi^{\prime}, D^{\prime}\right) v_{j}^{\nu \mu-1}\left(x^{\prime}, y^{\prime}, \xi^{\prime}\right), 1 \leqq k \leqq \theta^{\prime}, \\
& L_{e}\left(x^{\prime}, \nabla_{x^{\prime}} \tilde{\psi}\left(x^{\prime}, \xi^{\prime}\right)\right) \boldsymbol{v}_{e}^{\nu \mu}\left(x^{\prime}, y^{\prime}, \xi^{\prime}\right)=\delta_{\mu 0} G_{e}^{\nu}\left(x^{\prime}, y^{\prime}, \xi^{\prime}\right) \\
& \quad-Q_{e}^{1}\left(x^{\prime}, \xi^{\prime}, D^{\prime}\right) v_{\theta^{\prime}}^{\nu \mu}-Q_{e}^{2}\left(x^{\prime}, \xi^{\prime}, D^{\prime}\right) v_{e}^{\nu \mu-1}, \nu, \mu=0,1,2, \cdots,
\end{aligned}
$$

where $\boldsymbol{v}^{\nu,-1}\left(x^{\prime}, y^{\prime}, \xi^{\prime}\right) \equiv 0$. We can solve (2.25) and (2.26) with the initial conditions

$$
\begin{array}{r}
\left.\boldsymbol{v}^{\nu \mu}\left(x^{\prime}, y^{\prime}, \xi^{\prime}\right)\right|_{x_{1} \downarrow-\infty}=0\left(\left.\boldsymbol{v}^{\nu \mu}\right|_{x_{1}<x_{1}^{0}-\varepsilon_{0}} \equiv 0 \text { for some } \varepsilon_{0}\right), \\
\nu, \mu=0,1,2, \cdots .
\end{array}
$$

In fact, since (2.25) is a system of ordinary differential equations for $v_{k}^{\nu \mu}$ along rays corresponding to (2.1), we can determine $v_{k}^{\nu \mu}\left(x^{\prime}, y^{\prime}, \xi^{\prime}\right), 1 \leqq k \leqq l$, by (2.25) if $\boldsymbol{G}^{\nu}$ and $\boldsymbol{v}^{\nu \mu-1}$ are determined. Then $\boldsymbol{v}_{e}^{\nu \mu}\left(x^{\prime}, y^{\prime}, \xi^{\prime}\right)$ can be determined by (2.26). Thus $\boldsymbol{v}^{\nu}\left(x^{\prime}, y^{\prime}, \xi^{\prime}\right)$ is obtained from (2.24) which is justified in $\S 3$. (2.19) gives $\boldsymbol{a}^{\nu}\left(x^{\prime}, y^{\prime}, \xi^{\prime}\right)$ and, therefore, (2.6), (2. 7), (2.10) and (2.11) give $a_{j}^{\nu}\left(x, y^{\prime}, \xi^{\prime}\right), 1 \leqq j \leqq l_{1}$, and $c_{j}^{\nu \mu}\left(x, y^{\prime}, \xi\right)\left(1 \leqq j \leqq l-l_{1}, \mu=0,1, \cdots\right)$ if $a_{j}^{\nu-1}\left(x, y^{\prime}, \xi^{\prime}\right), 1 \leqq j \leqq l_{1}$, and $c_{j}^{\nu-1 \mu}\left(x, y^{\prime}, \xi\right)\left(1 \leqq j \leqq l-l_{1}, \mu=0,1, \cdots\right)$ are determined. From (2.14), (2.15), (2.17) and (2.23) we obtain $G^{\nu+1}$. Therefore the above arguments give the $a_{j}\left(x, y^{\prime}, \xi^{\prime}\right)$ and the $c_{j}\left(x, y^{\prime}, \xi\right)$ if (2.5), (2.9) and (2.14) are justified and if the $\boldsymbol{a}^{\nu}\left(x^{\prime}, y^{\prime}, \xi^{\prime}\right)$ defined by (2.19) are shown to satisfy (2.18).

\section{§ 3. Main Theorem}

A ray corresponding to (2.1) is a solution of the equations 


$$
\begin{aligned}
& d X^{\prime} / d s\left(s ; x^{\prime}, \xi^{\prime}\right)=\left(1,-\nabla_{\xi^{\prime \prime}} \xi_{1}\left(X^{\prime}\left(s ; x^{\prime}, \xi^{\prime}\right), \nabla_{x^{\prime \prime}} \tilde{\psi}\left(X^{\prime}, \xi^{\prime}\right)\right)\right) \\
& X^{\prime}\left(0 ; x^{\prime}, \xi^{\prime}\right)=x^{\prime}
\end{aligned}
$$

Then $X^{\prime}\left(s ; x^{\prime}, \xi^{\prime}\right)$ is a $C^{\infty}$ function, homogeneous of degree 0 in $\xi^{\prime}$ and defined when $x^{\prime}$ belongs to a small neighborhood of $x^{\prime \prime}$ and $\left(X^{\prime}\left(s ; x^{\prime}, \xi^{\prime}\right), \xi^{\prime}\right) \in \Gamma_{0}$. Now we can assume without loss of generality that

$$
\Gamma_{0}=U_{0} \times \gamma_{0}, U_{0}=\left\{x^{\prime} \in \boldsymbol{R}^{n} ;\left|x_{j}-x_{j}^{0}\right|<\varepsilon_{0}, 1 \leqq j \leqq n\right\}
$$

and $\gamma_{0}$ is a conic neighborhood of $\xi^{0^{\prime}}$ in $\boldsymbol{R}^{n} \backslash\{0\}$. Thus we can assume that $X^{\prime}\left(s ; x^{\prime}, \xi^{\prime}\right)$ is defined and $\left(X^{\prime}\left(s ; x^{\prime}, \xi^{\prime}\right), \xi^{\prime}\right) \in \Gamma_{0}$ when $\left(x^{\prime}, \xi^{\prime}\right) \in \Gamma_{1}$ and $x_{1}^{0}-\varepsilon_{0}-x_{1} \leqq s \leqq x_{1}^{0}+\varepsilon_{0}-x_{1}$, if necessary, shrinking $\Gamma_{1}$.

$T_{i j}\left(x^{\prime}, \xi^{\prime}\right)$ defined by (2.22) is homogeneous of degree $\rho_{j}-\rho_{i}$ and, therefore, $T_{i j}\left(x^{\prime}, \xi^{\prime}\right)$ belongs to a symbol class $S_{\rho_{j}-\rho_{i}}\left(\Gamma_{0}\right)$, i.e.,

$$
\begin{aligned}
\left|\partial_{x^{\prime}}^{\alpha^{\prime}} \partial_{\xi^{\prime}}^{\beta^{\prime}} T_{i j}\left(x^{\prime}, \xi^{\prime}\right)\right| \leqq C_{\alpha^{\prime} \beta^{\prime}}\left(1+\left|\xi^{\prime}\right|\right)^{\rho_{j}-\rho_{i}-\left|\beta^{\prime}\right|} & \\
& \text { when }\left(x^{\prime}, \xi^{\prime}\right) \in \Gamma_{0} \text { and }\left|\xi^{\prime}\right| \geqq 1 .
\end{aligned}
$$

In order to determine $\boldsymbol{v}_{\theta^{\prime}}^{\nu \mu}$ we consider the equations

$$
\begin{aligned}
& \left(D_{1}-\sum_{j=2}^{n} \partial \xi_{1} / \partial \zeta_{j}\left(x^{\prime}, \nabla_{x^{\prime \prime}} \tilde{\psi}\right) D_{j}\right) v_{k}\left(x^{\prime}, y^{\prime}, \xi^{\prime}\right)+\left(\xi_{1}-\xi_{1}\left(x^{0^{\prime}}, \xi^{\prime \prime}\right)\right) \\
& \quad \times v_{k}\left(x^{\prime}, y^{\prime}, \xi^{\prime}\right)+\sum_{j=1}^{\theta^{\prime}} T_{k j}\left(x^{\prime}, \xi^{\prime}\right) v_{j}\left(x^{\prime}, y^{\prime}, \xi^{\prime}\right) \\
& \quad=G_{0 k}\left(x^{\prime}, y^{\prime}, \xi^{\prime}\right)+G_{1 k}\left(x^{\prime}, y^{\prime}, \xi^{\prime}\right), \quad 1 \leqq k \leqq \theta^{\prime}, \\
& \left.v_{k}\left(x^{\prime}, y^{\prime}, \xi^{\prime}\right)\right|_{x_{1} \leqq x_{1}^{0}-\varepsilon_{0}} \equiv 0, \quad 1 \leqq k \leqq \theta^{\prime} .
\end{aligned}
$$

Lemma 3. 1. Assume that $G_{0 k}\left(x^{\prime}, y^{\prime}, \xi^{\prime}\right) \in S^{1-\rho_{k}-m_{1}-\nu}\left(\dot{\Gamma}_{0}\right)$ and

$$
\begin{aligned}
& G_{1 k}\left(x^{\prime}, y^{\prime}, \xi^{\prime}\right)=\int_{-\infty}^{0} \exp \left[i\left(\xi_{1}-\xi_{1}\left(x^{0^{\prime}}, \xi^{\prime \prime}\right)\right) s\right] g_{1 k}\left(x^{\prime}, y^{\prime}, \xi^{\prime}, s\right) d s, \\
& g_{1 k}\left(x^{\prime}, y^{\prime}, \xi^{\prime}, s\right) \in S^{1-\rho_{k}-m_{1}-\nu}\left(\dot{\Gamma}_{0} \times(-\infty, 0]\right),
\end{aligned}
$$

i.e., when $\left(x^{\prime}, y^{\prime}, \xi^{\prime}, s\right) \in \dot{\Gamma}_{0} \times(-\infty, 0]$ and $\left|\xi^{\prime}\right| \geqq 1$

$$
\left|\partial_{x^{\prime}}^{\alpha^{\prime}} \partial_{y^{\prime}}^{\beta^{\prime}} \partial_{\xi}^{r^{\prime}}, \partial_{s}^{j} g_{1 k}\left(x^{\prime}, y^{\prime}, \xi^{\prime}, s\right)\right| \leqq C_{\alpha^{\prime} \beta^{\prime} \gamma^{\prime} j}\left(1+\left|\xi^{\prime}\right|\right)^{1-\rho_{k}-m_{1}-\nu-\left|r^{\prime}\right|}
$$

and that supp $G_{0 k}\left(x^{\prime}, y^{\prime}, \xi^{\prime}\right) \subset \dot{\Gamma}_{1}$,

$$
g_{1 k}\left(x^{\prime}, y^{\prime}, \xi^{\prime}, s\right)=0 \text { if }\left(y^{\prime}, \xi^{\prime}\right) \notin \Gamma_{1} \text { or } s \leqq x_{1}^{0}-\varepsilon_{0}-x_{1},
$$

where $1 \leqq k \leqq \theta^{\prime}$. Then the solutions of the equations (3.2) and (3.3) can be written in the form 


$$
v_{k}\left(x^{\prime}, y^{\prime}, \xi^{\prime}\right)=\int_{-\infty}^{0} \exp \left[i\left(\xi_{1}-\xi_{1}\left(x^{0}, \xi^{\prime \prime}\right)\right) s\right] d_{k}\left(x^{\prime}, y^{\prime}, \xi^{\prime}, s\right) d s,
$$

where $d_{k}\left(x^{\prime}, y^{\prime}, \xi^{\prime}, s\right) \in S^{1-\rho_{k}-m_{1}-\nu}\left(\dot{\Gamma}_{0} \times(-\infty, 0]\right)$ and

$$
d_{k}\left(x^{\prime}, y^{\prime}, \xi^{\prime}, s\right)=0 \text { if }\left(y^{\prime}, \xi^{\prime}\right) \notin \Gamma_{1} \text { or } s \leqq x_{1}^{0}-\varepsilon_{0}-x_{1} .
$$

Proof. Along the ray defined by (3.1) (3.2) becomes the system of ordinary differential equations

$$
\begin{aligned}
& \frac{d v_{k}\left(X^{\prime}\left(s ; x^{\prime}, \xi^{\prime}\right), y^{\prime}, \xi^{\prime}\right)}{d s}+i\left(\xi_{1}-\xi_{1}\left(x^{0^{\prime}}, \xi^{\prime \prime}\right)\right) v_{k}\left(X^{\prime}, y^{\prime}, \xi^{\prime}\right) \\
& +i \sum_{j=1}^{\theta^{\prime}} T_{k j}\left(X^{\prime}, \xi^{\prime}\right) v_{j}\left(X^{\prime}, y^{\prime}, \xi^{\prime}\right)= \\
& \quad i G_{0 k}\left(X^{\prime}, y^{\prime}, \xi^{\prime}\right) \\
& +i G_{1 k}\left(X^{\prime}, y^{\prime}, \xi^{\prime}\right), \quad 1 \leqq k \leqq \theta^{\prime} .
\end{aligned}
$$

Since $\left.v_{k}\left(X^{\prime}\left(s ; x^{\prime}, \xi^{\prime}\right), y^{\prime}, \xi^{\prime}\right)\right|_{s \leqq x_{1}^{0}-\varepsilon_{0}-x_{1}}=0$, we have

$$
\begin{aligned}
& \boldsymbol{v}_{\theta^{\prime}}\left(X^{\prime}\left(s ; x^{\prime}, \xi^{\prime}\right), y^{\prime}, \xi^{\prime}\right)=i \int_{-\infty}^{s} d s^{\prime} \exp \left[-i \int_{s^{\prime}}^{s}\left\{\left(\xi_{1}-\xi_{1}\left(x^{0^{\prime}}, \xi^{\prime \prime}\right)\right) I_{\theta^{\prime}}\right.\right. \\
& \left.\left.\quad+T_{\theta^{\prime}}\left(X^{\prime}\left(q ; x^{\prime}, \xi^{\prime}\right), \xi^{\prime}\right)\right\} d q\right]\left\{\boldsymbol{G}_{0 \theta^{\prime}}\left(X^{\prime}\left(s^{\prime} ; x^{\prime}, \xi^{\prime}\right), y^{\prime}, \xi^{\prime}\right)\right. \\
& \left.\quad+G_{1 \theta^{\prime}}\left(X^{\prime}\left(s^{\prime} ; x^{\prime}, \xi^{\prime}\right), y^{\prime}, \xi^{\prime}\right)\right\}
\end{aligned}
$$

where $\boldsymbol{v}_{\theta^{\prime}}={ }^{t}\left(v_{1}, \cdots, v_{\theta^{\prime}}\right), \quad G_{j \theta^{\prime}}={ }^{t}\left(G_{j 1}, \cdots, \quad G_{j \theta^{\prime}}\right), j=0, \cdot 1, \quad$ and $T_{\theta^{\prime}}=$ $\left(T_{i j}\right)_{i, j=1, \ldots \theta^{\prime}}$ Thus putting $s=0$ in (3.4) we have

$$
\begin{aligned}
& \mathbb{E}_{\theta^{\prime}}\left(x^{\prime}, y^{\prime}, \xi^{\prime}\right)=i \int_{-\infty}^{0} d s \exp \left[i\left(\xi_{1}-\xi_{1}\left(x^{0^{\prime}}, \xi^{\prime \prime}\right)\right) s\right] \\
& \quad \times\left\{\exp \left[-i \int_{s}^{0} T_{\theta^{\prime}}\left(X^{\prime}\left(q ; x^{\prime}, \xi^{\prime}\right), \xi^{\prime}\right) d q\right] \boldsymbol{G}_{0 \theta^{\prime}}\left(X^{\prime}\left(s ; x^{\prime}, \xi^{\prime}\right), y^{\prime}, \xi^{\prime}\right)\right. \\
& +\int_{s}^{0} d s^{\prime} \exp \left[-i \int_{s^{\prime}}^{0} T_{\theta^{\prime}}\left(X^{\prime}\left(q ; x^{\prime}, \xi^{\prime}\right), \xi^{\prime}\right) d q\right] g_{1 \theta^{\prime}}\left(X^{\prime}\left(s^{\prime} ; x^{\prime}, \xi^{\prime}\right), y^{\prime},\right. \\
& \left.\left.\xi^{\prime}, s-s^{\prime}\right)\right\},
\end{aligned}
$$

where $g_{1 \theta^{\prime}}={ }^{t}\left(g_{11}, \cdots, g_{1 \theta^{\prime}}\right)$. On the other hand it is easily seen that if the $(i, j)$-entries of $M\left(x^{\prime}, \xi^{\prime}, s\right)$ are homogeneous of degree $\rho_{j}$ $-\rho_{i}$ in $\xi^{\prime}$ the $(i, j)$-entries of $\exp \left[M\left(x^{\prime}, \xi^{\prime}, s\right)\right]$ are homogeneous of degree $\rho_{j}-\rho_{i}$ in $\xi^{\prime}$. So the lemma easily follows.

Q. E. D.

Lemma 3. 2. (i) Let $v_{k}^{\nu \mu}\left(x^{\prime}, y^{\prime}, \xi^{\prime}\right)$ be solutions of (2. 25) - (2.27). Then we have for $1 \leqq k \leqq \theta^{\prime}$ 
(3.5) $\quad v_{k}^{\nu \mu}\left(x^{\prime}, y^{\prime}, \xi^{\prime}\right)=\int_{-\infty}^{0} \exp \left[i\left(\xi_{1}-\xi_{1}\left(x^{0^{\prime}}, \xi^{\prime \prime}\right)\right) s\right] d_{k}^{p \mu}\left(x^{\prime}, y^{\prime}, \xi^{\prime}, s\right) d s$,

(3.6) $\quad d_{k}^{\eta \mu}\left(x^{\prime}, y^{\prime}, \xi^{\prime}, s\right) \in S^{1-\rho_{k}-m_{1}-\nu-\mu}\left(\dot{\Gamma}_{0} \times(-\infty, 0]\right), \quad \nu, \mu=0,1,2, \cdots$.

For $\theta^{\prime}+1 \leqq k \leqq l$ we have

$$
\begin{aligned}
v_{k}^{\nu \mu}\left(x^{\prime}, y^{\prime}, \xi^{\prime}\right)= & v_{0 k}^{\nu \mu}\left(x^{\prime}, y^{\prime}, \xi^{\prime}\right)+\int_{-\infty}^{0} \exp \left[i\left(\xi_{1}-\xi_{1}\left(x^{0^{\prime}}, \xi^{\prime \prime}\right)\right) s\right] \\
& \times d_{k}^{\nu \mu}\left(x^{\prime}, y^{\prime}, \xi^{\prime}, s\right) d s
\end{aligned}
$$

(3. 8) $v_{0 k}^{\nu \mu}\left(x^{\prime}, y^{\prime}, \xi^{\prime}\right) \in S^{-\rho_{b}-m_{1}-\nu-\mu}\left(\dot{\Gamma}_{0}\right)$,

(3.9) $\quad d_{k}^{\mu \mu}\left(x^{\prime}, y^{\prime}, \xi^{\prime}, s\right) \in S^{-\rho_{k}-m_{1}-\nu-\mu}\left(\dot{\Gamma}_{0} \times(-\infty, 0]\right), \nu, \mu=0,1,2, \cdots$.

Moreover we have

$$
\operatorname{supp} v_{0 k}^{\nu \mu}\left(x^{\prime}, y^{\prime}, \xi^{\prime}\right) \subset \dot{\Gamma}_{1}
$$

$$
d_{k}^{\nu \mu}\left(x^{\prime}, y^{\prime}, \xi^{\prime}, s\right)=0 \text { if }\left(y^{\prime}, \xi^{\prime}\right) \notin \Gamma_{1} \text { or } s \leqq-\varepsilon_{1}+x_{1}^{0}-x_{1} \text {. }
$$

We can define $d_{k}^{v}\left(x^{\prime}, y^{\prime}, \xi^{\prime}, s\right) \in S^{\alpha_{k}-\rho_{k}-m_{1}-\nu}\left(\dot{\Gamma}_{0} \times(-\infty, 0]\right)$ and $v_{0 k}^{v}\left(x^{\prime}\right.$, $\left.y^{\prime}, \xi^{\prime}\right) \in S^{-\rho_{k}-m_{1}-\nu}\left(\dot{\Gamma}_{0}\right)$ by

$$
\begin{aligned}
& d_{k}^{\nu}\left(x^{\prime}, y^{\prime}, \xi^{\prime}, s\right) \sim \sum_{\mu=0}^{\infty} d_{k}^{\mu \mu}\left(x^{\prime}, y^{\prime}, \xi^{\prime}, s\right), \\
& v_{0 k}^{\nu}\left(x^{\prime}, y^{\prime}, \xi^{\prime}\right) \sim \sum_{\mu=0}^{\infty} v_{0 k}^{\nu \mu}\left(x^{\prime}, y^{\prime}, \xi^{\prime}\right),
\end{aligned}
$$

respectively, and put

$$
\begin{aligned}
v_{k}^{\nu}\left(x^{\prime}, y^{\prime}, \xi^{\prime}\right)= & v_{0 k}^{\nu}\left(x^{\prime}, y^{\prime}, \xi^{\prime}\right)+\int_{-\infty}^{0} \exp \left[i\left(\xi_{1}-\xi_{1}\left(x^{0^{\prime}}, \xi^{\prime \prime}\right)\right) s\right] \\
& \times d_{k}^{\nu}\left(x^{\prime}, y^{\prime}, \xi^{\prime}, s\right) d s,
\end{aligned}
$$

where $v_{0 k}^{\circ}\left(x^{\prime}, y^{\prime}, \xi^{\prime}\right) \equiv 0$ for $1 \leqq k \leqq \theta^{\prime}$ and $\alpha_{k}=1$ if $1 \leqq k \leqq \theta^{\prime},=0$ if $\theta^{\prime}+1 \leqq k \leqq l$.

(ii) Let $\tilde{a}_{k}^{\nu}\left(x^{\prime}, y^{\prime}, \xi^{\prime}\right), \tilde{c}_{k}^{\nu}\left(x^{\prime}, y^{\prime}, \xi^{\prime}\right)$ be defined by (2.16) and (2.19). For $1 \leqq k \leqq l_{1}$ we have

$$
\begin{aligned}
a_{k}^{u}\left(x, y^{\prime}, \xi^{\prime}\right)= & a_{0 k}^{y}\left(x, y^{\prime}, \xi^{\prime}\right)+\int_{-\infty}^{0} \exp \left[i\left(\xi_{1}-\xi_{1}\left(x^{0^{\prime}}, \xi^{\prime \prime}\right)\right) s\right] \\
& \times a_{1 k}^{u}\left(x, y^{\prime}, \xi^{\prime}, s\right) d s
\end{aligned}
$$

$$
a_{0 k}^{\nu}\left(x, y^{\prime}, \xi^{\prime}\right) \in S^{1-m_{1}-\nu}\left([0, \varepsilon) \times \dot{\Gamma}_{0}\right),
$$

where the $a_{k}^{\nu}\left(x, y^{\prime}, \xi^{\prime}\right)$ are the solutions of (2.5) and (2.6). 
Moreover

$$
\operatorname{supp} D^{\alpha} a_{0 k}^{\nu}\left(0, x^{\prime}, y^{\prime}, \xi^{\prime}\right) \subset \dot{\Gamma}_{1} \text { for any } \alpha \text {, }
$$

(iii) For $1 \leqq k \leqq l-l_{1}$

$$
\begin{aligned}
c_{k}^{\mu \mu}\left(x, y^{\prime}, \xi\right)= & c_{0 k}^{\nu \mu}\left(x, y^{\prime}, \xi\right)+\int_{-\infty}^{0} \exp \left[i\left(\xi_{1}-\xi_{1}\left(x^{0^{\prime}}, \xi^{\prime \prime}\right)\right) s\right] \\
& \times c_{1 k}^{\nu \mu}\left(x, y^{\prime}, \xi, s\right) d s
\end{aligned}
$$

$$
\begin{aligned}
& c_{0 k}^{\nu \mu}\left(x, y^{\prime}, \xi\right)=\sum_{\text {finite sump }} p\left(x, \xi_{0}, \nabla_{x^{\prime}} \tilde{\psi}\left(x^{\prime}, \xi^{\prime}\right)\right)^{-j} c_{0 k j}^{\nu \mu}\left(x, y^{\prime}, \xi\right), \\
& c_{0 k j}^{\nu \mu}\left(x, y^{\prime}, \xi\right) \in S^{1+j m-m_{1}-k-\nu-\mu}\left([0, \varepsilon) \times \dot{\Gamma}_{0} \times C\right)
\end{aligned}
$$

(with obvious definition),

(3. 22) $\quad c_{1 k}^{\nu \mu}\left(x, y^{\prime}, \xi, s\right)=\sum_{\text {finite } \operatorname{sum}} p\left(x, \xi_{0}, \nabla_{x^{\prime}} \tilde{\psi}\right)^{-j} c_{1 k j}^{\nu \mu}\left(x, y^{\prime}, \xi, s\right)$, (3. 23) $\quad c_{1 k j}^{\nu \mu}\left(x, y^{\prime}, \xi, s\right) \in S^{1+j m-m_{1}-k-\nu-\mu}\left([0, \varepsilon) \times \dot{\Gamma}_{0} \times C \times(-\infty, 0]\right)$, where the $c_{k}^{\nu \mu}\left(x, y^{\prime}, \xi\right)$ are defined by (2.10) and (2.11). Moreover

$$
\begin{aligned}
& \operatorname{supp} D^{\alpha} c_{0 k}^{\nu \mu}\left(0, x^{\prime}, \xi\right) \subset \Gamma_{1} \times C \text { for any } \alpha, \\
& c_{1 k}^{\nu \mu}\left(x, y^{\prime}, \xi, s\right)=0 \text { if }\left(y^{\prime}, \xi^{\prime}\right) \in \Gamma_{1} \text { or } s \leqq x_{1}^{0}-\varepsilon_{0}-x_{1} .
\end{aligned}
$$

Proof. By induction the lemma can be proved. From Lemma 3. 1, (2.15), (2.17), (2.23) and (2.25) it follows that (3.5), (3.6) and (3.11) are valid when $\nu, \mu=0,1 \leqq k \leqq \theta^{\prime}$. Then by (2.26) we have $(3.7)-(3.11)$, when $\nu, \mu=0, \theta^{\prime}+1 \leqq k \leqq l$. Let us assume that (3.5) - (3.11) hold for $\nu=\nu_{0}$ and $\mu=0$ and that (3.14) - (3.25) hold for $\nu=\nu_{0}-1$ and $\mu=0,1,2, \cdots$. By the induction assumptions, Lemma 3.1 , (2.25) and (2.26) we can inductively obtain (3.5)-(3.11) for $\nu=\nu_{0}$ and $\mu=1,2, \cdots$. Thus (3.12) and (3.13) can be interpreted as the asymptotic sums of symbols. Thus $\tilde{a}_{k}^{\nu_{0}}\left(x^{\prime}, y^{\prime}, \xi^{\prime}\right)$ and $\tilde{c}_{k}^{\nu_{0}}\left(x^{\prime}, y^{\prime}, \xi^{\prime}\right)$ can be defined by (2.16) and (2.19). Then the transport equations (2.6) with the boundary conditions (2.7) give (3.14)-(3.18) for $\nu=\nu_{0}$. From (2.10) and (2.11) (3.19)-(3.25) also follow. From Lemma 3.1, (2.14), (2.17), (2.23), (2.25) and (2.26) it follows that (3.5) $-(3.11)$ are valid when $\nu=\nu_{0}+1$ and $\mu=0$. Here we have used the fact that $f_{k}^{\nu_{0}+1}\left(x^{\prime}, y^{\prime}, \xi^{\prime}\right)$ can be determined by (2.14) as the sums of the asymptotic sums of symbols and the integrals of the 
asymptotic sums of symbols. So the induction argument is complete.

Q.E. D.

We can define $a_{0 k}\left(x, y^{\prime}, \xi^{\prime}\right) \in S^{1-m_{1}}\left([0, \varepsilon) \times \dot{\Gamma}_{0}\right)$ and $a_{1 k}\left(x, y^{\prime}, \xi^{\prime}, s\right)$ $\in S^{1-m_{1}}\left([0, \varepsilon) \times \dot{\Gamma}_{0} \times(-\infty, 0]\right)$ by $a_{0 k}\left(x, y^{\prime}, \xi^{\prime}\right) \sim \sum_{\nu=0}^{\infty} a_{0 k}^{\nu}\left(x, y^{\prime}, \xi^{\prime}\right)$ and $a_{1 k}\left(x, y^{\prime}, \xi^{\prime}, s\right) \sim \sum_{\nu=0}^{\infty} a_{1 k}^{v}\left(x, y^{\prime}, \xi^{\prime}, s\right)$, respectively. Put

$$
\begin{aligned}
& a_{k}^{\nu \mu}\left(x, y^{\prime}, \xi^{\prime}\right)=\frac{1}{2 \pi i} \int_{c_{\xi^{\prime}}} c_{k}^{\mu \nu}\left(x, y^{\prime}, \xi^{\prime}\right) \xi_{0}^{k-1} \exp \left[i x_{0} \xi_{0}\right] d \xi_{0} \\
& \quad \equiv a_{0 k}^{\nu \mu}\left(x, y^{\prime}, \xi^{\prime}\right)+\int_{-\infty}^{0} \exp \left[i\left(\xi_{1}-\xi_{1}\left(x^{0^{\prime}}, \xi^{\prime \prime}\right)\right) s\right] a_{1 k}^{\eta \mu}\left(x, y^{\prime}, \xi^{\prime}, s\right) d s .
\end{aligned}
$$

Then, $a_{0 k}^{y \mu}\left(x, y^{\prime}, \xi^{\prime}\right) \in S_{11}^{1-m_{1}-\nu-\mu}\left([0, \varepsilon) \times \dot{\Gamma}_{0}\right)$, i.e.,

$$
\begin{aligned}
& \left|\partial_{x}^{\alpha} \partial_{y^{\prime}}^{\beta^{\prime}} \partial_{\xi^{\prime}}^{\prime}, a_{0 k}^{y \mu}\left(x, y^{\prime}, \xi^{\prime}\right)\right| \leqq C_{\alpha \beta^{\prime} r^{\prime}}\left(1+\left|\xi^{\prime}\right|\right)^{1-m_{1}-\nu-\mu+\alpha_{0}-\left|r^{\prime}\right|} \\
& \text { when }\left(x, y^{\prime}, \xi^{\prime}\right) \in[0, \varepsilon) \times \dot{\Gamma}_{0} \text { and }\left|\xi^{\prime}\right| \geqq 1,
\end{aligned}
$$

and $a_{1 k}^{\nu \mu}\left(x, y^{\prime}, \xi^{\prime}, s\right) \in S_{11}^{1-m_{1}-\nu-\mu}\left([0, \varepsilon) \times \dot{\Gamma}_{0} \times(-\infty, 0]\right)$,

$$
a_{1 k}^{y \mu}\left(x, y^{\prime}, \xi^{\prime}, s\right)=0 \text { if }\left(y^{\prime}, \xi^{\prime}\right) \notin \Gamma_{1} \text { or } s \leqq x_{1}^{0}-\varepsilon_{0}-x_{1} .
$$

Therefore we can define $a\left(x, y^{\prime}, \xi^{\prime}\right)$ by

$$
\begin{aligned}
& a\left(x, y^{\prime}, \xi^{\prime}\right)=e_{0}\left(x, y^{\prime}, \xi^{\prime}\right)+\int_{-\infty}^{0} \exp \left[i\left(\xi_{1}-\xi_{1}\left(x^{0^{\prime}}, \xi^{\prime \prime}\right)\right) s\right] \\
& \times e_{1}\left(x, y^{\prime}, \xi^{\prime}, s\right) d s, \\
& e_{0}\left(x, y^{\prime}, \xi^{\prime}\right) \sim \sum_{k=1}^{l-l_{1}} \sum_{\nu, \mu=0}^{\infty} a_{0 k}^{\nu \mu}\left(x, y^{\prime}, \xi^{\prime}\right) \text {, } \\
& e_{1}\left(x, y^{\prime}, \xi^{\prime}, s\right) \sim \sum_{k=1}^{l-l_{1}} \sum_{\nu, \mu=0}^{\infty} a_{1 k}^{\eta \mu}\left(x, y^{\prime}, \xi^{\prime}, s\right) .
\end{aligned}
$$

Then $e_{0}\left(x, y^{\prime}, \xi^{\prime}\right) \in S_{11}^{1-m_{1}}\left([0, \varepsilon) \times \dot{\Gamma}_{0}\right), e_{1}\left(x, y^{\prime}, \xi^{\prime}, s\right) \in S_{11}^{1-m_{1}}\left([0, \varepsilon) \times \dot{\Gamma}_{0}\right.$ $\times(-\infty, 0])$,

$$
e_{1}\left(x, y^{\prime}, \xi^{\prime}, s\right)=0 \text { if }\left(y^{\prime}, \xi^{\prime}\right) \notin \Gamma_{1} \text { or } s \leqq x_{1}^{0}-\varepsilon_{0}-x_{1} .
$$

Moreover $e_{0}\left(x, y^{\prime}, \xi^{\prime}\right)$ and $e_{1}\left(x, y^{\prime}, \xi^{\prime}, s\right)$ belong to $S^{-\infty}$ when $x_{0}>0$. So we can define the distribution

$$
\begin{aligned}
& E_{1}(g)=\sum_{j=1}^{l_{1}}\left[\int \exp \left[i \phi_{j}\left(x, y^{\prime}, \xi^{\prime}\right)\right] a_{0 j}\left(x, y^{\prime}, \xi^{\prime}\right)(B g)\left(y^{\prime}\right) d y^{\prime} d \xi^{\prime}\right. \\
& \quad+\int d y^{\prime} d \xi^{\prime} \int_{-\infty}^{0} d s \exp \left[i\left\{\phi_{j}\left(x, y^{\prime}, \xi^{\prime}\right)+\left(\xi_{1}-\xi_{1}\left(x^{0^{\prime}}, \xi^{\prime \prime}\right) s\right\}\right]\right. \\
& \left.\quad \times a_{1 j}\left(x, y^{\prime}, \xi^{\prime}, s\right)(B g)\left(y^{\prime}\right)\right]+\int \exp \left[i\left(\tilde{\psi}\left(x^{\prime}, \xi^{\prime}\right)-\tilde{\psi}\left(y^{\prime}, \xi^{\prime}\right)\right)\right]
\end{aligned}
$$




$$
\begin{gathered}
\times e_{0}\left(x, y^{\prime}, \xi^{\prime}\right)(B g)\left(y^{\prime}\right) d y^{\prime} d \xi^{\prime}+\int d y^{\prime} d \xi^{\prime} \int_{-\infty}^{0} d s \exp \left[i \left\{\tilde{\psi}\left(x^{\prime}, \xi^{\prime}\right)\right.\right. \\
\left.\left.-\tilde{\phi}\left(y^{\prime}, \xi^{\prime}\right)+\left(\xi_{1}-\xi_{1}\left(x^{0^{\prime}}, \xi^{\prime \prime}\right)\right) s\right\}\right] e_{1}\left(x, y^{\prime}, \xi^{\prime}, s\right)(B g)\left(y^{\prime}\right), \\
g \in \mathscr{D}^{\prime}(U),
\end{gathered}
$$

as an oscillatory integral, where $U$ is a small neighborhood of $x^{0^{\prime}}$.

Definition 3. 3. Let $x^{\prime}=x^{\prime}\left(t ; y^{\prime}, \eta^{\prime}\right)$ and $\zeta^{\prime}=\zeta^{\prime}\left(t ; y^{\prime}, \eta^{\prime}\right)$ be the solutions of a system of the equations

$$
\begin{aligned}
& d x^{\prime} / d t=\left(1,-\nabla_{\zeta^{\prime \prime}} \xi_{1}\left(x^{\prime}, \zeta^{\prime \prime}\right)\right), \\
& d \zeta^{\prime} / d t=\nabla_{x^{\prime}} \xi_{1}\left(x^{\prime}, \zeta^{\prime \prime}\right), \\
& x^{\prime}=y^{\prime}, \zeta^{\prime}=\eta^{\prime} \text { and } \eta_{1}-\xi_{1}\left(y^{\prime}, \eta^{\prime \prime}\right)=0 \text { when } t=0 .
\end{aligned}
$$

Then the curves $\left\{\left(x^{\prime}\left(t ; y^{\prime}, \eta^{\prime}\right), \zeta^{\prime}\left(t ; y^{\prime}, \eta^{\prime}\right)\right) \in \Gamma_{0} ; t \in \mathbb{R}\right\}$ are said to be boundary null-bicharacteristic strips. Let $x=x_{j}\left(t ; y^{\prime}, \eta^{\prime}\right)$ and $\zeta=\zeta_{j}\left(t ; y^{\prime}, \eta^{\prime}\right), 1 \leqq j \leqq l_{1}$, be the solutions of a system of the equations

$$
\begin{aligned}
& d x / d t=\left(1,-\nabla_{\zeta^{\prime}} \lambda_{j}^{+}\left(x, \zeta^{\prime}\right)\right), \\
& d \zeta / d t=\nabla_{x} \lambda_{j}^{+}\left(x, \zeta^{\prime}\right), \\
& x_{0}=0, x^{\prime}=y^{\prime}, \zeta^{\prime}=\eta^{\prime} \text { and } \zeta_{0}=\lambda_{j}^{+}\left(0, y^{\prime}, \eta^{\prime}\right) \text { when } t=0 .
\end{aligned}
$$

Then the curves $\left\{\left(x_{j}\left(t ; y^{\prime}, \eta^{\prime}\right), \zeta_{j}\left(t ; y^{\prime}, \eta^{\prime}\right)\right) ; t \geqq 0\right\}, 1 \leqq j \leqq l_{1}$, are said to be outgoing null-bicharacteristic strips. Further we define

$$
\begin{aligned}
& C_{0}\left(\Gamma_{0}\right)=\left\{\left(x^{\prime}, \zeta^{\prime}, y^{\prime}, \eta^{\prime}\right) \in \Gamma_{0} \times \Gamma_{0} ;\left(x^{\prime}, \zeta^{\prime}\right)=\left(y^{\prime}, \eta^{\prime}\right) \text { or } x_{1}>y_{1}\right. \\
& \quad \text { and there exists a boundary null-bicharacteristic strip which } \\
& \left.\quad \text { contains both }\left(x^{\prime}, \zeta^{\prime}\right) \text { and }\left(y^{\prime}, \eta^{\prime}\right)\right\}, \\
& C_{j}\left(\Gamma_{0}\right)=\left\{\left(x, \zeta, y^{\prime}, \eta^{\prime}\right) \in\left(T^{*}((0, \varepsilon) \times U) \backslash 0\right) \times \Gamma_{0}\right. \text {; there exists an } \\
& \quad \text { outgoing null-bicharacteristic strip which contains both } \\
& \left.\quad(x, \zeta) \text { and }\left(0, y^{\prime}, \lambda_{j}^{+}\left(0, y^{\prime}, \eta^{\prime}\right), \eta^{\prime}\right)\right\}, 1 \leqq j \leqq l_{1} .
\end{aligned}
$$

Let us define wave front sets for $u \in C^{\infty}\left([0, \varepsilon) ; \mathscr{D}^{\prime}(U)\right)$. Since we can regard $u \in C^{\infty}\left([0, \varepsilon) ; \mathscr{D}^{\prime}(U)\right)$ as an element of $\mathscr{D}^{\prime}((0, \varepsilon) \times U)$ we can define $W F(u)$ for $u \in C^{\infty}\left([0, \varepsilon) ; \mathscr{D}^{\prime}(U)\right)$ by regarding $u$ as an element of $\mathscr{D}^{\prime}((0, \varepsilon) \times U)$.

Definition 3.4. For $u \in C^{\infty}\left([0, \varepsilon) ; \mathscr{D}^{\prime}(U)\right)$ we say that a point $\left(x^{1^{\prime}}, \xi^{1^{\prime}}\right)$ in $T^{*} U \backslash 0$ is not in the set $W F_{0}(u)$ if there exist $\phi \in C_{0}^{\infty}(U)$, 
a conic neighborhood $\gamma_{1}$ of $\xi^{1^{\prime}}$ and a positive constant $\varepsilon_{1}$ such that $\phi\left(x^{1 \prime}\right) \neq 0$ and

$$
\left|\mathscr{F}_{x^{\prime}}\left[\phi\left(x^{\prime}\right) D_{0}^{j} u(x)\right]\left(x_{0}, \xi^{\prime}\right)\right| \leqq C_{k j}\left(1+\left|\xi^{\prime}\right|\right)^{-k}
$$

when $\xi^{\prime} \in \gamma_{1}, x_{0} \in\left[0, \varepsilon_{1}\right)$ and $k, j=0,1,2, \cdots$.

The following lemma is an immediate consequence of the definition.

Lemma 3.5. Let $u \in C^{\infty}\left([0, \varepsilon) ; \mathscr{D}^{\prime}(U)\right)$.

(i) $\left(x^{1^{\prime}}, \xi^{1^{\prime}}\right) \notin W F_{0}(u)$ if and only if there exist a properly supported pseudo-differential operator in $x^{\prime}$ variables elliptic at ( $x^{1^{\prime}}$, $\left.\xi^{1^{\prime}}\right)$ and a positive constant $\varepsilon_{1}$ such that $A u\left(x_{0}, x^{\prime}\right) \in C^{\infty}\left(\left[0, \varepsilon_{1}\right) \times U\right)$.

(ii) $W F_{0}(A u) \subset W F_{0}(u)$ for every properly supported pseudodifferential operator in $x^{\prime}$ variables.

(iii) $\pi\left(W F_{0}(u)\right)=U \backslash\left\{x^{\prime} \in U\right.$; there exist a neighborhood $U_{1}$ of $x^{\prime}$ and a positive constant $\varepsilon_{1}$ such that $\left.u \in C^{\infty}\left(\left[0, \varepsilon_{1}\right) \times U_{1}\right)\right\}$, where $\pi$ : $T^{*} U \backslash 0 \rightarrow U$ is the natural projection, i.e., $\pi\left(x^{\prime}, \xi^{\prime}\right)=x^{\prime}$.

Now we can state our main theorem.

Theorem 3.6. Assume that the conditions $(A .1)-(A .3)$ are satisfied. Then $\left\{E_{1}, \Gamma,[0, \varepsilon) \times U\right\}$ is a right microlocal parametrix for the problem $(1.1)^{\prime}-(1.3)^{\prime}$ with $k=1$ at $\left(x^{0}, \xi^{\prime \prime}\right)$, where the ope rator $E_{1}$ is defined by (3.26) and $\varepsilon(>0)$ and $U$ are suitably chosen. Moreover we have

$$
\begin{aligned}
& W F\left(E_{1}(g)\right) \subset \cup_{j=1}^{l_{1}} C_{j}\left(\Gamma_{0}\right) \circ C_{0}\left(\Gamma_{0}\right) \circ W F(g)^{\dagger}, \\
& W F_{0}\left(E_{1}(g)\right) \subset C_{0}\left(\Gamma_{0}\right) \circ W F(g) \text { for } g \in \mathscr{D}^{\prime}(U)
\end{aligned}
$$

Remark. (i) It is obvious that we can construct microlocal parametrices for the problems $(1.1)^{\prime}-(1.3)^{\prime}, 2 \leqq k \leqq l$, at $\left(x^{0}, \xi^{0^{\prime}}\right)$ and that we have the estimates (3.30) and (3.31) where $E_{1}(g)$ is replaced by $E_{k}(g), 2 \leqq k \leqq l$. (ii) $C_{0}\left(\Gamma_{0}\right)$ is related to a boundary

$\dagger$ Let $A$ and $B$ be sets, $C$ a subset of $A \times B$ and $D$ a subset of $B$. Then we write $C \circ D$ $=\{a \in A$; there exists $b$ in $D$ such that $(\mathrm{a}, \mathrm{b})$ in $C\}$. 
wave.

Proof. Let $a_{j}\left(x^{\prime}, y^{\prime}, \xi^{\prime}\right), 1 \leqq j \leqq l$, be symbols in $S^{\nu_{1}-m_{j}}\left(\dot{\Gamma}_{0}\right)$ and $b_{j}\left(x^{\prime}, y^{\prime}, \xi^{\prime}, s\right), 1 \leqq j \leqq l$, symbols in $S^{\nu_{2}-m_{j}}\left(\dot{\Gamma}_{0} \times(-\infty, 0]\right)$ such that $b_{j}\left(x^{\prime}\right.$, $\left.y^{\prime}, \xi^{\prime}, s\right)=0$ when $s \leqq s_{0}$ for some $s_{0}<0$, and put $\boldsymbol{a}\left(x^{\prime}, y^{\prime}, \xi^{\prime}\right)={ }^{t}\left(a_{1}, \cdots\right.$, $\left.a_{l}\right), \boldsymbol{b}={ }^{t}\left(b_{1}, \cdots, b_{l}\right)$. Then it follows that

$$
\begin{aligned}
\boldsymbol{a}\left(x^{\prime}, y^{\prime}, \xi^{\prime}\right) & +\int_{-\infty}^{0} \exp \left[i\left(\xi_{1}-\xi_{1}\left(x^{0^{\prime}}, \xi^{\prime \prime}\right)\right) s\right] \boldsymbol{b}\left(x^{\prime}, y^{\prime}, \xi^{\prime}, s\right) d s \\
& \in S^{-\infty}\left(\dot{\Gamma}_{0} \times(-\infty, 0]\right)
\end{aligned}
$$

if

$$
\begin{aligned}
& \left(U\left(x^{\prime}, \nabla_{x^{\prime}} \tilde{\psi}\left(x^{\prime}, \xi^{\prime}\right)\right)+\sum_{k=1}^{n} \partial U / \partial \zeta_{k} \cdot D_{k}\right)\left\{\boldsymbol{a}\left(x^{\prime}, y^{\prime}, \xi^{\prime}\right)\right. \\
& \left.\quad+\int_{-\infty}^{0} \exp \left[i\left(\xi_{1}-\xi_{1}\left(x^{\prime \prime}, \xi^{\prime \prime}\right)\right) s\right] \boldsymbol{b}\left(x^{\prime}, y^{\prime}, \xi^{\prime}, s\right) d s\right\} \equiv 0\left(\bmod S^{-\infty}\right) .
\end{aligned}
$$

Thus the arguments in $\S 2$ and Lemma 3.2 show that $\left\{E_{1}, \Gamma,[0\right.$, $\varepsilon) \times U$ \} is a microlocal parametrix at $\left(x^{0}, \xi^{0^{\prime}}\right)$. Applying the method of stationary phase for (3.26) we obtain

$$
\begin{aligned}
& W F\left(E_{1}(g)\right) \subset \cup_{j=1}^{l_{1}} \Lambda_{j}^{\prime}\left(\Gamma_{0}\right) \circ W F(g), \\
& W F_{0}\left(E_{1}(g)\right) \subset \Lambda_{0}^{\prime}\left(\Gamma_{0}\right) \circ W F(g),
\end{aligned}
$$

where

$$
\begin{aligned}
& \Lambda_{j}^{\prime}\left(\Gamma_{0}\right)=\left\{\left(x, \zeta, y^{\prime}, \eta^{\prime}\right) \in\left(T^{*}((0, \varepsilon) \times U) \backslash 0\right) \times \Gamma_{0} ; \zeta=\nabla_{x} \phi_{j}\left(x, y^{\prime}, \xi^{\prime}\right),\right. \\
& \eta^{\prime}=-\nabla_{y^{\prime}} \phi_{j} \text { if } \nabla_{\xi^{\prime}} \phi_{j}=0 \text { for some }\left(y^{\prime}, \xi^{\prime}\right) \in \Gamma_{0} \text { or if } \xi_{1}= \\
& \xi_{1}\left(x^{0^{\prime}}, \xi^{\prime \prime}\right) \text { and } \nabla_{\xi^{\prime}} \phi_{j}\left(x, y^{\prime}, \xi^{\prime}\right)+s \nabla_{\xi^{\prime}}\left(\xi_{1}-\xi_{1}\left(x^{0^{\prime}}, \xi^{\prime \prime}\right)\right)=0 \text { for } \\
& \text { some } \left.\left(y^{\prime}, \xi^{\prime}\right) \in \Gamma_{0} \text { and } s<0\right\}, \\
& \Lambda_{0}^{\prime}\left(\Gamma_{0}\right)=\left\{\left(x^{\prime}, \zeta^{\prime}, y^{\prime}, \eta^{\prime}\right) \in \Gamma_{0} \times \Gamma_{0} ;\left(x^{\prime}, \zeta^{\prime}\right)=\left(y^{\prime}, \eta^{\prime}\right) \text { or } \zeta^{\prime}=\right. \\
& \nabla_{x^{\prime}} \tilde{\psi}\left(x^{\prime}, \xi^{\prime}\right), \eta^{\prime}=\nabla_{y^{\prime}} \tilde{\psi}\left(y^{\prime}, \xi^{\prime}\right) \text { if } \xi_{1}=\xi_{1}\left(x^{0^{\prime}}, \xi^{\prime \prime}\right) \text { and } \nabla_{\xi^{\prime}}\left(\tilde{\psi}\left(x^{\prime}, \xi^{\prime}\right)\right. \\
& \left.-\tilde{\psi}\left(y^{\prime}, \xi^{\prime}\right)\right)+s \nabla_{\xi^{\prime}}\left(\xi_{1}-\xi_{1}\left(x^{0^{\prime}}, \xi^{\prime \prime}\right)\right)=0 \text { for some }\left(y^{\prime}, \xi^{\prime}\right) \in \Gamma_{0} \text { and } \\
& s<0\} .
\end{aligned}
$$

It suffices to show that there exists a boundary null-bicharacteristic strip which contains both $\left(x^{\prime}, \zeta^{\prime}\right)$ and $\left(y^{\prime}, \eta^{\prime}\right)$ if $\zeta^{\prime}=\nabla_{x^{\prime}} \tilde{\psi}\left(x^{\prime}, \xi^{\prime}\right)$, $\eta^{\prime}=\nabla_{y^{\prime}} \tilde{\psi}\left(y^{\prime}, \xi^{\prime}\right), \xi_{1}=\xi_{1}\left(x^{0^{\prime}}, \xi^{\prime \prime}\right)$ and $\nabla_{\xi^{\prime}}\left(\tilde{\psi}\left(x^{\prime}, \xi^{\prime}\right)-\tilde{\psi}\left(y^{\prime}, \xi^{\prime}\right)\right)+s \nabla_{\xi^{\prime}}\left(\xi_{1}-\right.$ $\left.\xi_{1}\left(x^{\prime \prime}, \xi^{\prime \prime}\right)\right)=0$ for some $\left(y^{\prime}, \xi^{\prime}\right) \in \Gamma_{0}$ and $s<0$. For the same argument as in a proof of the above assertion yields characterizations of $\Lambda_{j}^{\prime}\left(\Gamma^{0}\right)$ and, therefore, one can prove the theorem. The uniqueness 
theorem for a system of ordinary differential equations and (2.1) imply that $\zeta^{\prime}\left(t ; y^{\prime}, \eta^{\prime}\right)=\nabla_{x^{\prime}} \tilde{\psi}\left(x^{\prime}\left(t ; y^{\prime}, \eta^{\prime}\right), \quad \xi^{\prime}\right)$ where $x^{\prime}\left(t ; y^{\prime}, \eta^{\prime}\right)$ and $\zeta^{\prime}\left(t ; y^{\prime}, \eta^{\prime}\right)$ are the solutions of (3.27)-(3.29). Thus we have

$$
\frac{d}{d t} \frac{\partial \tilde{\psi}}{\partial \xi_{j}}\left(x^{\prime}\left(t ; y^{\prime}, \eta^{\prime}\right), \xi^{\prime}\right)=\frac{\partial}{\partial \xi_{j}}\left(\xi_{1}-\xi_{1}\left(x^{0^{\prime}}, \xi^{\prime \prime}\right)\right), 1 \leqq j \leqq n .
$$

(3.32) yields

$$
\nabla_{\xi^{\prime}}\left\{\tilde{\psi}\left(x^{\prime}\left(t ; y^{\prime}, \eta^{\prime}\right), \xi^{\prime}\right)-\tilde{\phi}\left(y^{\prime}, \xi^{\prime}\right)\right\}=t \nabla_{\xi^{\prime}}\left(\xi_{1}-\xi_{1}\left(x^{0^{\prime}}, \xi^{\prime \prime}\right)\right) .
$$

Thus by (3.33) with $t=-s$ we have

$$
\nabla_{\xi^{\prime}} \tilde{\psi}\left(x^{\prime}, \xi^{\prime}\right)=\nabla_{\xi^{\prime}} \tilde{\psi}\left(x^{\prime}\left(-s ; y^{\prime}, \eta^{\prime}\right), \xi^{\prime}\right) \text {. }
$$

Since we may assume that $\operatorname{det}\left(\partial^{2} \tilde{\psi} / \partial x_{j} \partial \xi_{k}\left(x^{\prime}, \xi^{\prime}\right)\right)_{j, k=1, \ldots, n} \neq 0$ for $\left(x^{\prime}\right.$, $\left.\xi^{\prime}\right) \in \Gamma_{0}$ we obtain $x^{\prime}=x^{\prime}\left(-s ; y^{\prime}, \eta^{\prime}\right)$ and $\zeta^{\prime}\left(-s ; y^{\prime}, \eta^{\prime}\right)=\nabla_{x^{\prime}} \tilde{\psi}\left(x^{\prime}, \xi^{\prime}\right)$ $=\zeta^{\prime}$.

Q. E. D.

\section{§4. Microlocal Parametrices and Uniqueness Theorem}

It follows from Duistermaat and Hörmander [3] that there exists an operator $F: \mathscr{D}^{\prime}((-\varepsilon, \varepsilon) \times U) \rightarrow \mathscr{D}^{\prime}((-\varepsilon, \varepsilon) \times U)$ such that (i) $P F(f)-f \in C^{\infty}((-\varepsilon, \varepsilon) \times U)$ if $W F(f) \subset T^{*}\left(\left(-\varepsilon_{1}, \varepsilon_{1}\right) \times U_{1}\right) \backslash 0$, (ii) $W F(F(f)) \subset\left\{x_{1} \geqq c\right\}$ if $W F(f) \subset\left\{x_{1} \geqq c\right\}$, where $\varepsilon_{1}<\varepsilon$ and $U_{1}(\subset U)$ is a neighborhood of $x^{0^{\prime}}$ in $\mathbb{R}^{n}$. We write

$$
\mathscr{D}^{\prime}([0, \varepsilon) \times U)=\left\{f \in \mathscr{D}^{\prime}((-\varepsilon, \varepsilon) \times U) ; \operatorname{supp} f \subset[0, \varepsilon) \times U\right\} .
$$

$C^{\infty}\left[(0, \varepsilon) ; \mathscr{D}^{\prime}(U)\right)$ can be mapped injectively into $\mathscr{D}^{\prime}([0, \varepsilon) \times U)$ in a natural manner:

$$
C^{\infty}\left([0, \varepsilon) ; \mathscr{D}^{\prime}(U)\right) \ni f \rightarrow \tilde{f} \in \mathscr{D}^{\prime}([0, \varepsilon) \times U) .
$$

From partial hypoellipticity we can regard $\left.F(\tilde{f})\right|_{x_{0} \geq 0}$ as an element of $C^{\infty}\left([0, \varepsilon) ; \mathscr{D}^{\prime}(U)\right)$ if $f \in C^{\infty}\left([0, \varepsilon) ; \mathscr{D}^{\prime}(U)\right)$. Thus we can define maps $W_{j}^{0}: C^{\infty}\left([0, \varepsilon) ; \mathscr{D}^{\prime}(U)\right) \rightarrow \mathscr{D}^{\prime}(U)$ by

$$
W_{j}^{0}(f)=\lim _{x_{0} \rightarrow+0} B_{j}\left(x^{\prime}, D\right) F(\tilde{f})\left(x_{0}, x^{\prime}\right), 1 \leqq j \leqq l .
$$

Put

$$
\begin{aligned}
E^{0}\left(f, g_{1}, \cdots, g_{l}\right)=\left.F(\tilde{f})\right|_{x_{0} \geqq 0}+\sum_{j=1}^{l} E_{j}\left(g_{j}-W_{j}^{0}(f)\right) \\
\quad \text { for } f \in C^{\infty}\left([0, \varepsilon) ; \mathscr{D}^{\prime}(U)\right), \quad g_{j} \in \mathscr{D}^{\prime}(U), \quad 1 \leqq j \leqq l .
\end{aligned}
$$


Then we have the following

Theorem 4.1. Under the conditions $(A .1)-(A .3)$ the above operator $E^{0}: C^{\infty}\left([0, \varepsilon) ; \mathscr{D}^{\prime}(U)\right) \times\left\{\mathscr{D}^{\prime}(U)\right\}^{l} \rightarrow C^{\infty}\left([0, \varepsilon) ; \mathscr{D}^{\prime}(U)\right)$ satisfies the following properties:

(i ) $P E^{0}\left(f, g_{1}, \cdots, g_{l}\right)-f \in C^{\infty}([0, \varepsilon) \times U)$ if $W f(\tilde{f}) \subset T^{*}\left(\left(-\varepsilon_{1}, \varepsilon_{1}\right) \times U_{1}\right) \backslash 0$.

(ii) $\left.B_{j} E^{0}\left(f, g_{1}, \cdots, g_{l}\right)\right|_{x_{0}=0}-g_{j} \in C^{\infty}(U)$ if $W F_{0}(f), W F\left(g_{j}\right) \subset \Gamma, 1 \leqq j \leqq l$, and $W F(f) \subset\left(0, \varepsilon_{1}\right) \times U_{1}$ $\times \mathbb{R} \times \boldsymbol{\gamma}_{1}$.

(iii) $W F\left(E^{0}\left(f, g_{1}, \cdots, g_{l}\right)\right) \subset\left\{x_{1} \geqq c\right\}$ if $W F(f) \subset\left\{x_{1} \geqq c\right\}$ and $W F_{0}(f), W F\left(g_{j}\right) \subset\left\{x_{1} \geqq c\right\}$.

Here $\varepsilon_{1}(<\varepsilon)$ is a small positive constant and $U_{1} \times \gamma_{1}(\subset \Gamma)$ is a small conic neighborhood of $\left(x^{0^{\prime}}, \xi^{0^{\prime}}\right)$. Moreover we have

$$
\begin{gathered}
W F\left(E^{0}\left(f, g_{1}, \cdots, g_{l}\right)\right) \subset \widetilde{C} \circ W F(f) \cup\left[\cup_{j=1}^{l_{1}} C_{j}\left(\Gamma_{0}\right) \circ C_{0}\left(\Gamma_{0}\right) \circ\right. \\
\left.\left\{\bigcup_{k=1}^{l} W F\left(g_{k}\right) \cup\left(\widetilde{C}_{\imath} \circ W F(f)\right) \cup W F_{0}(f)\right\}\right], \\
W F_{0}\left(E^{\circ}\left(f, g_{1}, \cdots, g_{l}\right)\right) \subset C_{0}\left(\Gamma_{0}\right) \circ\left[\cup_{j=1}^{l} W F\left(g_{j}\right) \cup\left(\widetilde{C}_{\partial} \circ W F(f)\right)\right. \\
\left.\cup W F_{0}(f)\right],
\end{gathered}
$$

where

$$
\begin{aligned}
& \widetilde{C}=\left\{(x, \xi, y, \eta) \in\left(T^{*}((0, \varepsilon) \times U) \backslash 0\right)^{2} ;(x, \xi)=(y, \eta) \text { or } x_{1}>y_{1}\right. \\
& \quad \text { and there exists a null bicharacteristic strip for } p \text { which } \\
& \quad \text { contains both }(x, \xi) \text { and }(y, \eta)\}, \\
& \widetilde{C}_{\partial}=\left\{\left(x^{\prime}, \xi^{\prime}, y, \eta\right) \in\left(T^{*} U \backslash 0\right) \times\left(T^{*}((0, \varepsilon) \times U) \backslash 0\right) ; x_{1}>y_{1}\right. \text { and } \\
& \quad \text { there exists a null bicharacteristic strip for } p \text { which contains } \\
& \left.\quad \text { both }\left(0, x^{\prime}, \xi_{0}, \xi^{\prime}\right) \text { and }(y, \eta) \text { for some } \xi_{0} \in \mathbb{R}\right\} .
\end{aligned}
$$

Let us construct a microlocal parametrix for the problem (1.1) - (1.3) in the case where $f$ belongs to $\mathscr{D}^{\prime}([0, \varepsilon) \times U)$, following Melrose [6]. We assume in the remainder of this section that (A. 4) $\left\{B_{j}\left(x^{\prime}, D\right)\right\}_{1 \leqq j \leqq l}$ is a normal system on the hyperplane $x_{0}=0$ and $m_{j} \leqq m-1,1 \leqq j \leqq l$.

Therefore we obtain a Dirichlet system $\left\{B_{j}\right\}_{1 \leqq j \leqq m}$ of order $m$ on $x_{0}=0$ by complementing suitably $\left\{B_{\jmath}\right\}_{l+1 \leqq j \leqq m}$. 
Lemma 4.2([5], [8], [9]). There exists a Dirichlet system $\left\{B_{j}^{\prime}\left(x^{\prime}, D\right)\right\}_{1 \leq j \leq m}$, uniquely determined, such that for any $u \in C^{\infty}([0$, $\left.\varepsilon) ; \mathscr{D}^{\prime}(U)\right)$

$$
P \tilde{u}-(P u)^{\sim}=\sum_{j=1}^{m} B_{j}^{\prime}\left(x^{\prime}, D\right)\left\{\left.\delta\left(x_{0}\right) \otimes B_{j}\left(x^{\prime}, D\right) u(x)\right|_{x_{0}=0}\right\} .
$$

Moreover we have

$$
\begin{aligned}
i^{m-l} & \operatorname{det}\left(\frac{1}{2 \pi i} \oint \frac{b_{j}^{\prime}\left(x^{\prime}, \xi_{0},-\xi^{\prime}\right) \xi_{0}^{k-1}}{p_{-}\left(0, x^{\prime},-\xi_{0}, \xi^{\prime}\right)} d \xi_{0}\right)_{\substack{j=l+1, \ldots, \ldots \\
k=1, \ldots, m-l}} \\
= & (\operatorname{sgn} \sigma)(-1)^{l(l-1) / 2} b_{1}\left(x^{\prime}, 1,0, \cdots, 0\right)^{-1} \ldots b_{m}^{-1} \\
& \quad \times \operatorname{det}\left(\frac{1}{2 \pi i} \oint \frac{b_{j}\left(x^{\prime}, \xi\right) \xi_{0}^{k-1}}{p_{+}\left(0, x^{\prime}, \xi\right)} d \xi_{0}\right)_{j, k=1, \ldots, l} \text { Im } \xi_{1} \leqq 0,
\end{aligned}
$$

where $b_{j}^{\prime}$ is the principal part of $B_{j}^{\prime}, \sigma$ is a permutation defined by $m_{o(j)}=j-1,1 \leqq j \leqq m$, and $p_{-}(x,-\xi)=\prod_{j=1}^{m-l}\left(\xi_{0}-\lambda_{j}^{-}\left(x,-\xi^{\prime}\right)\right)$, Im $\xi_{1} \geqq 0$.

Put

$$
\begin{aligned}
& \mathscr{M}=\left\{f \in \mathscr{D}^{\prime}([0, \varepsilon) \times U) ; f=\sum_{j=l+1}^{m} B_{j}^{\prime}\left(x^{\prime}, D\right)\left(\delta\left(x_{0}\right) \otimes h_{j}\left(x^{\prime}\right)\right),\right. \\
&\left.h_{j} \in \mathscr{D}^{\prime}(U)\right\}, \\
& \mathscr{M}_{1}=\left\{f \in \mathscr{D}^{\prime}([0, \varepsilon) \times U) ; f=\sum_{j=0}^{m-1} \delta^{(\jmath)}\left(x_{0}\right) \otimes h_{j}\left(x^{\prime}\right), h_{j} \in C^{\infty}(U)\right\}, \\
& \dot{C}^{\infty}([0, \varepsilon) \times U)=\left\{\tilde{f} \in \mathscr{D}^{\prime}([0, \varepsilon) \times U) ; f \in C^{\infty}([0, \varepsilon) \times U)\right\} .
\end{aligned}
$$

Lemma 4.3. $\mathscr{M}$ is uniquely determined by $\left\{P, B_{1}, \cdots, B_{l}\right\}$.

Proof. Assume that $\left\{B_{1}, \cdots, B_{l}, \tilde{B}_{l+1}, \cdots, \tilde{B}_{m}\right\}$ is another Dirichlet system of order $m$ on $x_{0}=0$. Then we have

$$
\begin{aligned}
\sum_{j=1}^{m} B_{j}^{\prime}\left(x^{\prime}, D\right)\left(\left.\delta\left(x_{0}\right) \otimes B_{j}\left(x^{\prime}, D\right) u(x)\right|_{x_{0}=0}\right) \\
=\sum_{j=1}^{l} \tilde{B}_{j}^{\prime}\left(x^{\prime}, D\right)\left(\left.\delta\left(x_{0}\right) \otimes B_{j} u\right|_{x_{0}=0}\right)+\sum_{j=l+1}^{m} \tilde{B}_{j}^{\prime}\left(x^{\prime}, D\right)\left(\delta\left(x_{0}\right)\right. \\
\left.\left.\quad \otimes \tilde{B}_{j} u\right|_{x_{0}=0}\right)
\end{aligned}
$$

for $u \in C^{\infty}\left([0, \varepsilon) ; \mathscr{D}^{\prime}(U)\right)$, where $\left\{\tilde{B}_{j}^{\prime}\right\}_{1 \leq j \leq m}$ is defined for $\left\{B_{1}, \cdots\right.$, $\left.B_{l}, \tilde{B}_{l+1}, \cdots, \tilde{B}_{m}\right\}$ by Lemma 4.2. On the other hand it easily follows that for any $g_{j} \in \mathscr{D}^{\prime}(U), 1 \leqq j \leqq m$, there exists $u$ in $C^{\infty}([0, \varepsilon)$; $\left.\mathscr{D}^{\prime}(U)\right)$ such that $\left.B_{j}\left(x^{\prime}, D\right) u(x)\right|_{x_{0}=0}=g_{j}\left(x^{\prime}\right), 1 \leqq j \leqq m$ (see [5]). This proves the lemma.

Q. E. D.

Definition 4.5. Let $u \in \mathscr{D}^{\prime}([0, \varepsilon) \times U)$. We define $W F_{1}(u)$ by 


$$
\begin{aligned}
W F_{1}(u)= & \left\{\left(x^{\prime}, \xi^{\prime}\right) \in T^{*} U \backslash 0 ; \iota^{*-1}(\gamma) \cap W F(u) \neq \varnothing\right. \text { for any } \\
& \text { conic neighborhood } \left.\gamma \text { of }\left(x^{\prime}, \xi^{\prime}\right)\right\},
\end{aligned}
$$

where the inclusion $\iota: U \rightarrow(-\varepsilon, \varepsilon) \times U$ is defined by $\iota\left(x^{\prime}\right)=\left(0, x^{\prime}\right)$.

Definition 4. 6. A right microlocal parametrix for the problems $(1.1)-(1.3)$ at $\left(x^{0}, \xi^{\prime \prime}\right)$ is a triple $\left\{E^{1}, \Gamma,[0, \varepsilon) \times U\right\}$ satisfying the conditions

(i) $\Gamma$ is a conic neighborhood of $\left(x^{0^{\prime \prime}}, \xi^{\prime \prime}\right), U$ is a neighborhood of $x^{0^{\prime}}$ and $\varepsilon>0$,

(ii) $E^{1}$ is an operator: $\mathscr{D}^{\prime}([0, \varepsilon) \times U) \times\left\{\mathscr{D}^{\prime}(U)\right\}^{l} \rightarrow \mathscr{D}^{\prime}([0, \varepsilon) \times U)$,

(iii) $P E^{1}\left(f, g_{1}, \cdots, g_{l}\right)-f-\sum_{j=1}^{l} B_{j}^{\prime}\left(x^{\prime}, D\right)\left(\delta\left(x_{0}\right) \otimes g_{j}\left(x^{\prime}\right)\right) \in C^{\infty}([0$, $\varepsilon) \times U)+\mathscr{M}+\mathscr{M}_{1}$ if $W F_{1}(f), W F\left(g_{j}\right) \subset \Gamma$ and $W F\left(\left.f\right|_{x_{0}>0}\right) \subset\left(0, \varepsilon_{1}\right) \times$ $U_{1} \times \boldsymbol{R} \times \gamma_{1}$, where $\Gamma=U_{1} \times \gamma_{1}$ and $\gamma_{1}$ is a conic neighborhood of $\xi^{0^{\prime}}$,

(iv) $W F\left(\left.E^{1}\left(f, g_{1}, \cdots, g_{l}\right)\right|_{x_{0}>0}\right) \subset\left\{x_{1} \geqq c\right\}$ if $W F\left(\left.f\right|_{x_{0}>0}\right) \subset\left\{x_{1} \geqq c\right\}$ and $W F_{1}(f), W F\left(g_{j}\right) \subset\left\{x_{1} \geqq c\right\}$.

It follows from partial hypoellipticity that we can define maps $W_{j}^{1}: \mathscr{D}^{\prime}([0, \varepsilon) \times U) \rightarrow \mathscr{D}^{\prime}(U)$ by

$$
W_{j}^{1}(f)=\lim _{x_{0} \rightarrow-0} B_{j}\left(x^{\prime}, D\right) F(f)\left(x_{0}, x^{\prime}\right) .
$$

Put

$$
\begin{aligned}
E^{1}\left(f, g_{1}, \cdots, g_{l}\right)= & F(f)-\left(\left.F(f)\right|_{x_{0} \leqq 0}\right) \sim \sum_{j=1}^{l}\left(E_{j}\left(g_{j}-W_{j}^{1}(f)\right)\right)^{\sim} \\
& \text { for } f \in \mathscr{D}^{\prime}([0, \varepsilon) \times U), g_{j} \in \mathscr{D}^{\prime}(U),
\end{aligned}
$$

where $\left(\left.F(f)\right|_{x_{0} \leqq 0}\right)^{\sim} \in \mathscr{D}^{\prime}((-\varepsilon, 0] \times U)$ is defined for $\left.F(f)\right|_{x_{0} \leqq 0} \in$ $C^{\infty}\left((-\varepsilon, 0] ; \mathscr{D}^{\prime}(U)\right)$ in a natural manner. Then we have the following

Theorem 4.7. Assume that the conditions (A.1)-(A.4) are satisfied. Then modifying $U, \Gamma$ and $\varepsilon$, if necessary, $\left\{E^{1}, \Gamma,[0, \varepsilon)\right.$ $\times U\}$ is a microlocal parametrix for $(1.1)-(1.3)$ at $\left(x^{0}, \xi^{0^{\prime}}\right)$. Moreover we have

$$
\begin{gathered}
W F\left(\left.E^{1}\left(f, g_{1}, \cdots, g_{l}\right)\right|_{x_{0}>0}\right) \subset \widetilde{C}_{0} \circ F\left(\left.f\right|_{x_{0}>0}\right) \cup\left[\cup_{j=1}^{l_{1}} C_{j}\left(\Gamma_{0}\right) \circ\right. \\
\left.C_{0}\left(\Gamma_{0}\right) \circ\left\{\bigcup_{k=1}^{l} W F\left(g_{k}\right) \cup\left(\widetilde{C}_{\partial} \circ W F\left(\left.f\right|_{x_{0}>0}\right)\right) \cup W F_{1}(f)\right\}\right], \\
W F_{1}\left(E^{1}\left(f, g_{1}, \cdots, g_{l}\right)\right) \subset C_{0}\left(\Gamma_{0}\right) \circ\left[\bigcup_{j=1}^{l} W F\left(g_{j}\right) \cup\left(\widetilde{C}_{\partial} \circ W F\left(\left.f\right|_{x_{0}>0}\right)\right)\right. \\
\left.\cup W F_{1}(f)\right] .
\end{gathered}
$$


Using the result obtained by Lax and Nirenberg [7] the same argument as in [6], $\S 11$, yields the following

Theorem 4. 8. Let $\left(x^{1^{\prime}}, \xi^{1^{\prime}}\right) \in \Gamma=U_{1} \times \gamma_{1}$ and $u \in \mathscr{D}^{\prime}([0, \varepsilon) \times U)$. Then we have $\left(x^{1^{\prime}}, \xi^{1^{\prime}}\right) \notin W F_{1}(u)$ if there exists $v$ in $\mathscr{M}$ such that $\left(x^{1^{\prime}}, \xi^{1^{\prime}}\right) \notin W F_{1}(P u-v)$ and

$$
\begin{aligned}
\left(x^{1^{\prime}}, \xi^{1^{\prime}}\right) \notin C_{0}\left(\Gamma_{0}\right) \circ\left[\widetilde{C}_{\partial} \circ\left\{W F\left(\left.u\right|_{x_{0}>0}\right) \cap(0, \varepsilon) \times U_{1} \times \boldsymbol{R} \times \gamma_{1}\right\}\right. \\
\\
\left.\cup\left\{\left(W F_{1}(u) \backslash\left\{\left(x^{1^{\prime}}, \xi^{1^{\prime}}\right)\right\}\right) \cap \Gamma\right\}\right] .
\end{aligned}
$$

Corollary 4.9. Let $u \in C^{\infty}\left([0, \varepsilon) ; \mathscr{D}^{\prime}(U)\right)$. Then we have $\left(x^{1^{\prime}}\right.$, $\left.\xi^{1^{\prime}}\right) \notin W F_{0}(u)$ if $\left(x^{1^{\prime}}, \xi^{1^{\prime}}\right) \notin W F_{0}(P u),\left(x^{1^{\prime}}, \xi^{1^{\prime}}\right) \notin W F\left(\left.B_{J} u\right|_{x_{0}=0}\right), 1 \leqq j \leqq l$, and

$$
\begin{aligned}
\left(x^{1^{\prime}}, \xi^{1^{\prime}}\right) \notin C_{0}\left(\Gamma_{0}\right) \circ\left[\widetilde{C}_{\diamond} \circ\left\{W F(u) \cap(0, \varepsilon) \times U_{1} \times \boldsymbol{R} \times \gamma_{1}\right\}\right. \\
\left.\left.\cup\left\{W F_{0}(u) \backslash\left\{\left(x^{1^{\prime}}, \xi^{1^{\prime}}\right)\right\}\right) \cap \Gamma\right\}\right] .
\end{aligned}
$$

\section{§5. Some Remarks}

Let $\mathscr{E}:\left\{\mathscr{D}^{\prime}(U)\right\}^{l} \rightarrow C^{\infty}\left([0, \varepsilon) ; \mathscr{D}^{\prime}(U)\right)$ be a microlocal parametrix for the Dirichlet problem

$$
\begin{aligned}
& P(x, D) u(x)=0, \quad x \in \mathbb{R}_{+}^{n+1}, \quad x_{1}>0, \\
& \left.D_{1}^{k-1} u(x)\right|_{x_{1}=0}=0, \quad x_{0}>0, \quad 1 \leqq k \leqq m, \\
& \left.D_{0}^{j-1} u(x)\right|_{x_{0}=0}=h_{j}\left(x^{\prime}\right), \quad x_{1}>0, \quad 1 \leqq j \leqq l
\end{aligned}
$$

at $\left(x^{0}, \xi^{\prime \prime}\right)$. The operator $\mathscr{E}$ can be constructed in the same way as in $\S 2$. The phase functions $\phi_{j}\left(x, y^{\prime}, \xi^{\prime}\right)$ are defined by the eiconal equations

$$
\begin{aligned}
& \partial_{0} \phi_{j}\left(x, y^{\prime}, \xi^{\prime}\right)=\lambda_{j}^{+}\left(x, \nabla_{x^{\prime}} \phi_{j}\right), \\
& \phi_{j}\left(0, x^{\prime}, y^{\prime}, \xi^{\prime}\right)=\left(x^{\prime}-y^{\prime}\right) \cdot \xi^{\prime}, \quad 1 \leqq j \leqq l .
\end{aligned}
$$

Then we have the following

Lemma 5.1. Under the condition (A. 1) a microlocal parametrix for the Dirichlet problem can be given in the form

$$
\mathscr{E}\left(h_{1}, \cdots, h_{l}\right)=\sum_{k=1}^{l} \sum_{j=1}^{l_{1}} \int \exp \left[i \phi_{j}\left(x, y^{\prime}, \xi^{\prime}\right)\right] a_{j k}\left(x, y^{\prime}, \xi^{\prime}\right)
$$




$$
\begin{aligned}
& \times h_{k}\left(y^{\prime}\right) d y^{\prime} d \xi^{\prime}+\sum_{k=1}^{l} \int \exp \left[i\left(x^{\prime}-y^{\prime}\right) \cdot \xi^{\prime}\right] a_{k}\left(x, y^{\prime}, \xi^{\prime}\right) h_{k}\left(y^{\prime}\right) d y^{\prime} d \xi^{\prime}, \\
& \left(a_{1 k}^{0}\left(0, x^{\prime}, y^{\prime}, \xi^{\prime}\right), \cdots, a_{l_{1} k}^{0}, c_{1 k}^{0}\left(0, x^{\prime}, y^{\prime}, \xi^{\prime}\right), \cdots, c_{l-l_{1} k}^{0}\right)_{k \downarrow 1, \ldots l} \\
& =\chi\left(x^{\prime}, y^{\prime}, \xi^{\prime}\right)^{t} L_{d}\left(x^{\prime}, \xi^{\prime}\right)^{-1}
\end{aligned}
$$

where $a_{j_{k}}$ and $a_{k}$ are symbols, $a_{j k}^{0}$ and $a_{k}^{0}$ are their principal symbols as multiple symbols,

$$
\begin{aligned}
a_{k}^{0}\left(x, y^{\prime}, \xi^{\prime}\right)= & \sum_{j=1}^{l-l_{1}} \frac{1}{2 \pi i} \int_{c_{\xi^{\prime}}} p(x, \xi)^{-1} \xi_{0}^{j-1} \exp \left[i x_{0} \xi_{0}\right] d \xi_{0} \\
& \times c_{j k}^{0}\left(x, y^{\prime}, \xi^{\prime}\right)
\end{aligned}
$$

and

$$
\begin{aligned}
& L_{d}\left(x^{\prime}, \xi^{\prime}\right)=\left(\lambda_{1}^{+}\left(0, x^{\prime}, \xi^{\prime}\right)^{j-1}, \cdots, \lambda_{l_{1}}^{+j-1},\right. \\
& \left.-\frac{1}{2 \pi i} \int_{c_{\xi^{\prime}}} p\left(0, x^{\prime}, \xi\right)^{-1} \xi_{0}^{j-1} d \xi_{0}, \cdots, \frac{1}{2 \pi i} \int_{C_{\xi^{\prime}}} p^{-1} \xi_{0}^{j+l-l_{1}-2} d \xi_{0}\right)_{j \downarrow 1, \ldots, l} .
\end{aligned}
$$

Here we note that

$$
\begin{aligned}
\operatorname{det} L_{d}\left(x^{\prime}, \xi^{\prime}\right)= & (-1)^{\left(l / 2+l_{1}\right)(l-1)} \Pi_{1 \leqq j<k \leqq l_{1}}\left(\lambda_{j}^{+}\left(0, x^{\prime}, \xi^{\prime}\right)-\lambda_{k}^{+}\right) \\
& \times \Pi_{l_{1}+1 \leqq j \leqq l 1 \leqq k \leqq m-l}\left(\lambda_{j}^{+}-\lambda_{k}^{-}\right)^{-1} .
\end{aligned}
$$

Lemma 5. 2. Assume that the condition (A.1) is satisfied. For $h_{k} \in \mathscr{D}^{\prime}(U), 1 \leqq k \leqq l$, we can define pseudo-differential operators $\mathscr{B}_{j k}$ in $x^{\prime}$ variables by

$$
\sum_{k=1}^{l} \mathscr{B}_{j k}\left(h_{k}\right)=\left.B_{j}\left(x^{\prime}, D\right) \mathscr{E}\left(h_{1}, \cdots, h_{l}\right)\right|_{x_{0}=0} .
$$

Moreover the principal symbols $\beta_{j k}^{0}$ of $\mathscr{B}_{j k}$ can be given in the form

$$
\beta_{j k}^{0}\left(x^{\prime}, y^{\prime}, \xi^{\prime}\right)=\chi\left(x^{\prime}, y^{\prime}, \xi^{\prime}\right) L\left(x^{\prime}, \xi^{\prime}\right) L_{d}\left(x^{\prime}, \xi^{\prime}\right)^{-1} .
$$

Under the conditions (A. 1) - (A. 3) we can construct a microlocal parametrix $\mathscr{A}=\left(\mathscr{A}_{j k}\right)_{j, k=1, \ldots, l}$ for the problem

$$
\sum_{k=1}^{l} \mathscr{B}_{j k}\left(h_{k}\right)=g_{j}\left(x^{\prime}\right), g_{j} \in \mathscr{D}^{\prime}(U), 1 \leqq j \leqq l .
$$

Thus putting $E_{k}(g)=\mathscr{E}\left(\mathscr{A}_{1 k}(g), \cdots, \mathscr{A}_{l k}(g)\right)$, we obtain a right microlocal parametrix for the problem $(1.1)^{\prime}-(1.3)^{\prime}$ at $\left(x^{0}, \xi^{0^{\prime}}\right)$. 


\section{References}

[1] Chazarain, J., Construction de la paramétrix du problème mixte hyperbolique pour l'équation des ondes, G. R. Acad. Sci. Paris, 276 (1973), 1213-1215.

[2] Duff, G. F.D., On wave fronts, and boundary waves, Comm. Pure Appl. Math., 17 (1964), 189-225.

[3] Duistermaat, J. J. and Hörmander, L., Fourier integral operators, II, Acta Math., 128 (1972), 183-269.

[4] Ikawa, M., Problèmes mixtes pas nécessairement $L^{2}$-bien posés pour les équations strictement hyperboliques, Osaka J. Math., 12 (1975), 69-115.

[5] Lions, J. L. and Magenes, E., Problemes aux Limites non Homogenes et Applications, I, Dunod, Paris, 1968.

[6] Melrose, R. B., Microlocal parametrices for diffractive boundary value problems, Duke Math. J., 42 (1975), 605-635.

[7] Nirenberg, L., Lectures on Linear Differential Equations, Regional Conference Series in Mathematics, 20 (1973), Amer. Math. Soc., Providence. Rhode Island.

[ 8 ] Sakamoto, R., $\mathscr{E}$-well posedness for hyperbolic mixed problems with constant coefficients, J. Math. Kyoto Univ., 14 (1974), 93-118.

[9] Schechter, M., General boundary value problems for elliptic differential equations, Comm. Pure Appl. Math., 12 (1959), 457-486.

[10] Taylor, M. S., Grazing rays and reflection of singularities of solutions to wave equations, Comm. Pure Appl. Math., 29 (1976), 1-38.

[11] Wakabayashi, S., Singularities of the Riemann functions of hyperbolic mixed problems in a quarter-space, Publ. RIMS, Kyoto Univ., 11 (1976), 417-440.

[12] - Analytic wave front sets of the Riemann functions of hyperbolic mixed problems in a quarter-space, Publ. RIMS, Kyoto Univ., 11 (1976), 785-807. 
